\title{
Bivariate extension of the moment projection method for the particle population balance dynamics
}

\author{
Shaohua $\mathrm{Wu}^{\mathrm{a}}$, Casper Lindberg ${ }^{\mathrm{b}}$, Jethro Akroyd ${ }^{\mathrm{b}}$, Wenming Yang ${ }^{\mathrm{a}}$, Markus \\ Kraft*b,c \\ ${ }^{a}$ Department of Mechanical Engineering, National University of Singapore, \\ Engineering Block EA, Engineering Drive 1, Singapore, 117576 \\ ${ }^{b}$ Department of Chemical Engineering and Biotechnology, University of Cambridge, \\ West Cambridge Site, Philippa Fawcett Drive, Cambridge, CB3 OAS United Kingdom \\ ${ }^{c}$ School of Chemical and Biomedical Engineering, Nanyang Technological University, \\ 62 Nanyang Drive, Singapore, 637459 \\ corresponding author* \\ E-mail:mk306@cam.ac.uk
}

\begin{abstract}
This work presents a bivariate extension of the moment projection method (BVMPM) for solving the two-dimensional population balance equations involving particle inception, growth, shrinkage, coagulation and fragmentation. A two-dimensional Blumstein and Wheeler algorithm is proposed to generate a set of weighted particles that approximate the number density function. With this algorithm, the number of the smallest particles can be directly tracked, closing the shrinkage and fragmentation moment source terms. The performance of BVMPM has been tested against the hybrid method of moments (HMOM) and the stochastic method. Results suggest that BVMPM can achieve higher accuracy than HMOM in treating shrinkage and fragmentation processes where the number of the smallest particles plays an important role.
\end{abstract}

Keywords: bivariate; moment projection method; population balance 
dynamics

\section{Introduction}

2 The modeling of discrete populations of particles has found wide applica3 tions in environmental, biological, medical and technological systems [1-9].

4 The evolution of the particle population can be modeled using a population 5 balance equation (PBE), which can be expressed as the number density func6 tion (NDF) associated to the particles' properties [10]. In general, the NDF 7 depends on time, location and a set of internal coordinates such as particle 8 volume, temperature, composition and surface area. The PBEs usually con9 tain an inception term corresponding to the formation of particles from the

surrounding environment, a growth term due to particle surface reactions, a shrinkage term due to oxidation or evaporation, a coagulation term due to the collision and sticking of particles as well as a fragmentation term describing the breakage of large particles. The resulting PBE is mathematically an integro-differential equation which is so complex that analytical solution rarely exits.

For years, different numerical methods have been proposed to solve the PBEs. A review of the models of particle formation and the numerical methods used to solve them can be found in [11]. These methods often encompass a trade-off between accuracy and computational efficiency. The stochastic methods $[12,13]$ are able to provide a highly detailed description about the evolution of the NDF; however, under certain condition, the computational time and memory requirement can be intractable. In sectional methods [1417], the NDF is discretised into a number of sections or bins, then the PBE 
is transformed into a set of ordinary differential equations (ODEs) that describe the evolution of particle populations within each section. Sectional methods are intuitive. However, they usually require large numbers of sections to achieve high accuracy, making them computationally expensive. The method of moments (MOM) [18] enables a good balance between the physical details and computational efficiency. MOM is a class of methods for tracking a few lower-order moments from a population of particles without having explicit knowledge of the NDF itself as only the integral quantities of the particles are of interest for most applications. Unfortunately, the moment equations are usually unclosed. Depending on the coagulation kernel used, fractional-order moments may be present in the moment equations. These moments are not directly solver for and should be properly estimated. For the particle negative growth processes such as shrinkage and fragmentation, the number of the smallest particles is needed to close the corresponding moment equations. However, this information is lost in MOM since the NDF has been transformed into moments. Up to now, numerous methods have been introduced trying to handle these closure problems.

A successful approach to approximate the fractional-order moments is the method of moments with interpolative closure (MOMIC) [19-22] where a functional relationship between the fractional-order moments and integerorder moments is created. The formalism of MOMIC allows one to resolve for the number of the smallest particles for particle inception, growth and coagulation without closure problems. Because of numerical simplicity and ease of implementation, MOMIC has been widely adopted for the treatment of inception, coagulation and growth processes. Another closure approach 
is the quadrature method of moments (QMOM) [23-26] where the NDF is approximated using a set of weighted particles and weights which are computed by a product-difference (PD) algorithm [27] based on the moments. The direct quadrature method of moments (DQMOM) [28] is an extension of QMOM, where the particles and weights are tracked directly without referring to the PD algorithm. DQMOM has advantages of being computationally cheap and can be easily extended to describe multivariate PBEs. However, it suffers from the problem of singularities with certain initial conditions and artificial perturbations are needed to prevent failure in the numerical solution. Recently, the standard QMOM has been modified by applying the Gauss-Radau quadrature interpolation rule to fix one quadrature node at the smallest particle size. The resulting method, namely QMOM-Radau [29], leads to a better statistical representation of the PSD compared with the standard QMOM.

In order to handle the particle negative growth problem, a number of moment methods are proposed with the focus being on the reconstruction of the NDF [30-35]. In [30] a finite-size domain complete set of trial functions method of moments (FCMOM) is proposed where the NDF is approximated with a series of Legendre polynomials. Unfortunately, this method fails to guarantee the positivity of the reconstructed NDF due to the limited number of polynomials that can be determined. In the extended quadrature method of moments (EQMOM) [31, 32], the NDF is approximated with a set of continuous non-negative kernel density functions such as gamma, beta and log-normal functions. With the reconstructed NDF, the closure of the shrinkage or fragmentation moment equations becomes straightforward. However, 
this method requires prior information of the shape of the NDF to select a suitable kernel function.

Most of the methods described above are restricted to the univariate NDF, making them not suitably to include enough characteristics to accurately describe a nanoparticle system. For many applications, it is usually inefficient to describe the population of particles based on only one internal coordinate. For example, the soot particles formed in flames usually exist in the form of aggregates. A proper description of the soot particle population is usually based on a bivarate NDF that is a function of both the particle volume and surface area so that the fractal dimension can be considered. In most particle synthesis reactors, not only are the particle sizes evolving in time and location, but also is the particle morphology as a result of coagulation (also referred to as aggregation). To better design such reactors, it is necessary to adopt a mathematical description of the bivariate PBE which is more complex and computationally difficult.

As a historical footnote, in [36] the bivariate extension of MOM for the evolution of the two radii of curvature of ellipsoidal particles in a continuously fed batch reactor is considered for the first time. However, they did not actually complete a bivariate moment calculation but outlined a possible solution, i.e., using a large number of mixed moments, for the overly restrictive special case. In [37] a bivariate QMOM is proposed for modeling the dynamics of a population of inorganic nanoparticles undergoing simultaneous coagulation and particle sintering. The authors introduced two quadrature techniques, a multiple 3-point quadrature technique and a 12-point quadrature technique, to determine the particle positions and weights. The performance of the 
bivariate QMOM has been assessed by comparison with the high resolution discrete method, and it has exhibited high accuracy. However, this method is restricted to the calculation of specified number of moments. Furthermore, the 12-point quadrature technique requires the aid of the conjugate-gradient minimization algorithm which can be very difficult and computationally demanding. In [38] the QMOM is extended for solving two-dimensional batch crystallization models involving crystals nucleation, size-dependent growth, aggregation and dissolution. The authors have applied the orthogonal polynomials of lower-order moments to place the weighted particles. With this technique, one can calculate as many moments as required. However, this method is still restricted by the conjugate-gradient minimization algorithm. In [39], a conditional quadrature method of moments (CQMOM) was proposed. With this method, the multivariate NDF is rewritten as a product of univariate marginal NDF and a conditional NDF, both of which can be represented with a set of weighted particles. CQMOM has been successfully applied to simulations for TiO2-distributions [40], flash nanoprecipitation [41] and soot formation [42]. However, similar to QMOM, CQMOM cannot handle the shrinkage or fragmentation problem. In [29], a joint extended conditional quadrature method of moments (ECQMOM) is proposed which combines the technique of EQMOM and CQMOM. This method has been applied to simulate the soot formation process in a burner-stabilized premixed ethylene flame. The results are found to be in good agreement with the Monte Carlo results, suggesting the high accuracy of ECQMOM. In [43], a hybrid method of moments (HMOM) is introduced to simulate the soot formation in premixed flames and counter diffusion flames where the soot NDF 
is given based on particle volume and surface area. HMOM is a combination of DQMOM and MOMIC. It adopts the interpolation technique to approximate the fractional-order moments due to the application of realistic collision kernels. The soot NDF is discretised into two modes: the smallest particles and large particles. A source term for the smallest particles is proposed to close the shrinkage and fragmentation moment equations [44]. The resulting HMOM is mathematically simple, easy to implement and numerically robust.

Recently, a moment projection method (MPM) $[45,46]$ has been proposed. This method retains the advantages of ease of implementation and robustness, and at the same time it is able to directly track the number of the smallest particles. The performance of MPM for treating the particle shrinkage and fragmentation processes has been evaluated under different conditions and it is of great accuracy. In this work, we extend the MPM into a bivariate method (BVMPM) for solving the two-dimensional PBE including particle inception, growth, shrinkage, coagulation and fragmentation. The paper is organized as follows. Section 2 presents the moment methods for solving the bivariate particle population balance equations. The detailed mathematical formulation of BVMPM and the related algorithms are introduced. In section 3, the proposed BVMPM is compared with HMOM and the stochastic method for all the particle processes under different conditions. In section 4, principal conclusions are summarized. 


\section{Model formulation}

\subsection{Population balance equation}

For BVMPM, an important consideration is the realisability of the moment set. Realisability is related with the existence of an underlying NDF that corresponds to the moment set. If the set of moments are not realisable, they lead to unphysical distributions or no NDF can be described by such moments. The generation of unrealisable moments is usually caused due to the improper treatment of the spatial transportation of moments [47]. This problem can be avoided by properly designing the numerical schemes. In [48], a high-order-volume-scheme is proposed to guarantee the moment realisability for quadrature-based moment methods. The general idea behind this scheme is to evaluate the moment flux terms at the faces of the cells through interpolation of the weighted particles rather than the moments, thus preventing the realisability problem. In light of realisability, in this work we restrict our attention to the moment closure method for a bivariate particle system. The aim is to evaluate the BVMPM error in isolation. Therefore we simulate a spatially homogenous PBE with no moment spatial transportation terms. The obtained moments always remain realisable during the simulation time span. For the application of BVMPM to the spatially inhomogeneous particle systems, the realisable finite-volume numerical scheme can be adopted to gurantee the moment realisability. The spatially homogenous PBE governing the evolution of the bivariate particle distribution is given as follows:

$$
\frac{\mathrm{d} N(t ; i, j)}{\mathrm{d} t}=R(t ; i, j)+W(t ; i, j)+S(t ; i, j)+G(t ; i, j)+F(t ; i, j),
$$


where $N(t ; i, j)$ is the number of particles as a function of time $t$ and internal size coordinates $(i, j)$ which we will refer to as $N(i, j)$ from hereon. $R, W, S$, $G$ and $F$ are the inception, growth, shrinkage, coagulation and fragmentation source terms, respectively. The specific functional forms used in this work are as follows:

$$
\begin{aligned}
R\left(t ; i_{0}, j_{0}\right)= & K_{\mathrm{In}}, \\
W(t ; i, j)= & K_{\mathrm{G}}\left(N\left(i-\delta_{i}, j-\delta_{j}\right)-N(i, j)\right), \\
S(t ; i, j)= & K_{\mathrm{Sk}}\left(N\left(i+\delta_{i}, j+\delta_{j}\right)-N(i, j)\right), \\
G(t ; i, j)= & \frac{1}{2} \sum_{i^{\prime}=i_{0}}^{i} \sum_{j^{\prime}=j_{0}}^{j} K_{\mathrm{Cg}} N\left(i-i^{\prime}, j-j^{\prime}\right) N\left(i^{\prime}, j^{\prime}\right) \\
& -\sum_{i^{\prime}=i_{0}}^{\infty} \sum_{j^{\prime}=j_{0}}^{\infty} K_{\mathrm{Cg}} N(i, j) N\left(i^{\prime}, j^{\prime}\right), \\
F(t ; i, j)= & \sum_{i^{\prime}=i}^{\infty} \sum_{j^{\prime}=j}^{\infty} K_{\mathrm{Fg}}\left(i^{\prime}, j^{\prime}\right) P\left(i, j \mid i^{\prime}, j^{\prime}\right) N\left(i^{\prime}, j^{\prime}\right)-K_{\mathrm{Fg}}(i, j) N(i, j),
\end{aligned}
$$

where $K_{\text {In }}$ is the inception kernel that describes the formation rate of the particles at the smallest size coordinates $\left(i_{0}, j_{0}\right) . K_{\mathrm{G}}$ and $K_{\mathrm{Sk}}$ are the growth and shrinkage kernels, respectively. $\delta_{i}$ and $\delta_{j}$ refer to the change of the particle sizes in a single growth or shrinkage event. $K_{\mathrm{Cg}}$ is the coagulation kernel that describes the rate at which particles collide and stick together. Lastly, $K_{\mathrm{Fg}}(i, j)$ is the fragmentation kernel that describes the frequency with which particles fragment. The particles at the smallest sizes are not supposed to fragment, otherwise it may lead to an infinite number of particles of zero size and for this reason the total particle size would not be conserved $[49,50]$. As a result, the fragmentation kernel has to meet the following requirement: 


$$
K_{\mathrm{Fg}}(i, j)= \begin{cases}0, & \text { if } i<2 i_{0} \text { or } j<2 j_{0}, \\ K_{\mathrm{Fg}}, & \text { otherwise }\end{cases}
$$

$P\left(i, j \mid i^{\prime}, j^{\prime}\right)$ is the fragmentation distribution function which represents the number of particles at size coordinates $(i, j)$ formed by the fragmentation of particles at size coordinates $\left(i^{\prime}, j^{\prime}\right)$. Different types of fragmentation exist, such as symmetric fragmentation, erosion fragmentation, uniform fragmentation and parabolic fragmentation. This work only considers the erosion fragmentation. The application of BVMPM to other types of fragmentation can be implemented in a similar way. During an erosion event, one particle with the size coordinate $(i, j)$ breaks up into two fragments with one fragment having the minimum size $\left(i_{0}, j_{0}\right)$ and the other is of $\left(i-i_{0}, j-j_{0}\right)$. The fragmentation distribution function is described as:

$$
P\left(i, j \mid i^{\prime}, j^{\prime}\right)= \begin{cases}1 & \text { if } i=i_{0} \text { and } j=j_{0} \\ 1 & \text { if } i=i^{\prime}-i_{0} \text { and } j=j^{\prime}-j_{0} \\ 0 & \text { otherwise }\end{cases}
$$

The evaluations of the moment souce terms are dependent on these kernel functions. If realistic additive kernels or free-molecular Brownian kernels are used, fractional-order moments are present, which can be estimated by using either the interpolation technique as in MOMIC or the weighted particles as in QMOM. However, this will introduce an interpolation error. Since the aim here is to investigate the BVMPM error in isolation, constant kernels are adopted in this work. 
199

200

The $x$-th, $y$-th order moment $M_{x, y}$ of the bivariate NDF is given by:

$$
M_{x, y}=\sum_{i=i_{0}}^{\infty} \sum_{j=j_{0}}^{\infty} i^{x} j^{y} N(i, j) .
$$

201 Multiplying this expression with the PBE gives the bivariate moment evolu202 tion equation:

$$
\frac{\mathrm{d} M_{x, y}}{\mathrm{~d} t}=R_{x, y}(M)+W_{x, y}(M)+S_{x, y}(M, N)+G_{x, y}(M)+F_{x, y}(M, N)
$$

203 The moment source terms are as follows: 


$$
\begin{aligned}
& R_{x, y}(M)=K_{\text {In }} i_{0}^{x} j_{0}^{y}, \\
& W_{x, y}(M)=K_{\mathrm{G}} \sum_{m=0}^{x} \sum_{n=0}^{y}\left(\begin{array}{l}
x \\
m
\end{array}\right)\left(\begin{array}{l}
y \\
n
\end{array}\right) \delta_{i}^{x-m} \delta_{j}^{y-n} M_{m, n}-K_{\mathrm{G}} M_{x, y}, \\
& S_{x, y}(M, N)=K_{\mathrm{Sk}} \sum_{m=0}^{x} \sum_{n=0}^{y}\left(\begin{array}{l}
x \\
m
\end{array}\right)\left(\begin{array}{l}
y \\
n
\end{array}\right)\left(-\delta_{i}\right)^{x-m}\left(-\delta_{j}\right)^{y-n} M_{m, n}-K_{\mathrm{Sk}} M_{x, y} \\
& -K_{\mathrm{Sk}} \sum_{j=j_{0}}^{\infty} \sum_{i=i_{0}}^{i_{0}+\delta_{i}-1}\left(i-\delta_{i}\right)^{x}\left(j-\delta_{j}\right)^{y} N_{i, j} \\
& -K_{\mathrm{Sk}} \sum_{i=i_{0}}^{\infty} \sum_{j=j_{0}}^{j_{0}+\delta_{j}-1}\left(i-\delta_{i}\right)^{x}\left(j-\delta_{j}\right)^{y} N_{i, j} \\
& +K_{\mathrm{Sk}} \sum_{i=i_{0}}^{i_{0}+\delta_{i}-1} \sum_{j=j_{0}}^{j_{0}+\delta_{j}-1}\left(i-\delta_{i}\right)^{x}\left(j-\delta_{j}\right)^{y} N_{i, j}, \\
& G_{x, y}(M)=\frac{1}{2} K_{\mathrm{Cg}} \sum_{m=0}^{x} \sum_{n=0}^{y}\left(\begin{array}{l}
x \\
m
\end{array}\right)\left(\begin{array}{l}
y \\
n
\end{array}\right) M_{m, n} M_{x-m, y-n}-K_{\mathrm{Cg}} M_{x, y} M_{0,0}, \\
& F_{x, y}(M, N)=K_{\mathrm{Fg}} \sum_{m=0}^{x} \sum_{n=0}^{y}\left(\begin{array}{l}
x \\
m
\end{array}\right)\left(\begin{array}{l}
y \\
n
\end{array}\right)\left(-i_{0}\right)^{x-m}\left(-j_{0}\right)^{y-n} M_{m, n}+K_{\mathrm{Fg}} i_{0}^{x} j_{0}^{y} M_{0,0}-K_{\mathrm{Fg}} M_{x, y} \\
& -K_{\mathrm{Fg}} \sum_{j=j_{0}}^{\infty} \sum_{i=i_{0}}^{2 i_{0}-1}\left(\left(i-i_{0}\right)^{x}\left(j-j_{0}\right)^{y}+i_{0}^{x} j_{0}^{y}-i^{x} j^{y}\right) N_{i, j} \\
& -K_{\mathrm{Fg}} \sum_{i=i_{0}}^{\infty} \sum_{j=j_{0}}^{2 j_{0}-1}\left(\left(i-i_{0}\right)^{x}\left(j-j_{0}\right)^{y}+i_{0}^{x} j_{0}^{y}-i^{x} j^{y}\right) N_{i, j} \\
& +K_{\mathrm{Fg}} \sum_{i=i_{0}}^{2 i_{0}-1} \sum_{j=j_{0}}^{2 j_{0}-1}\left(\left(i-i_{0}\right)^{x}\left(j-j_{0}\right)^{y}+i_{0}^{x} j_{0}^{y}-i^{x} j^{y}\right) N_{i, j}
\end{aligned}
$$

204 The detailed derivations of these moment source terms can be found in Ap- 
pendix Appendix A. Since constant kernels are adopted, the moment source terms for growth and coagulation are closed by themselves. For shrinkage, however, the numbers of particles at the smallest size coordinates are needed to evaluate the particle boundary flux terms represented by the last three terms on the right-hand side of Eq. 13. Similarly, the accumulation of particles at the smallest sizes in fragmentation also requires the knowledge on the number of the smallest particles, as can be seen from Eq. 15. This is challenging to MOM since the detailed information on NDF has been lost when it is transformed into moments. Therefore, proper approximation on the numbers of the smallest particles has to be made to close these source terms.

\subsection{Bivariate moment projection method}

The general idea behind BVMPM is to rewrite the $\operatorname{NDF} N(i, j)$ as a product of a univariate marginal NDF $N(i)$ and a conditional NDF $N(j \mid i)$ :

$$
N(i, j)=N(i) N(j \mid i)
$$

As a result, the $x$-th, $y$-th order moment can be expressed as:

$$
M_{x, y}=\sum_{i=i_{0}}^{\infty} i^{x} N(i)\left(\sum_{j=j_{0}}^{\infty} j^{y} N(j \mid i)\right) .
$$

220 We define $M_{x, 0}=\sum_{i=i_{0}}^{\infty} i^{x} N(i)$ as the marginal moment and $M_{y \mid i}=\sum_{j=j_{0}}^{\infty} j^{y} N(j \mid i)$ as the conditional moment which meets:

$$
M_{0 \mid i}=\sum_{j=j_{0}}^{\infty} N(j \mid i)=1 .
$$




$$
\frac{\mathrm{d} \widetilde{M}_{x, y}}{\mathrm{~d} t}=R_{x, y}(\widetilde{M})+W_{x, y}(\widetilde{M})+S_{x, y}(\widetilde{M}, \widetilde{N})+G_{x, y}(\widetilde{M})+F_{x, y}(\widetilde{M}, \widetilde{N})
$$

where $\left(\alpha_{k}, \beta_{l \mid k}\right)$ are the internal size coordinates for the weighted particle. In order to evaluate the number of the smallest particles present in the shrinkage and fragmentation moment source terms, we fix one particle size, $\alpha_{1}$, to be located at the smallest size: $\alpha_{1}=i_{0}$. Given each $\alpha_{k}, \beta_{1 \mid k}$ is fixed at $j_{0}$ : $\beta_{1 \mid k}=j_{0}$. As a result, the pointwise values of the NDF at the smallest size coordinates can be evaluated. The $x$-th, $y$-th order empirical moment in BVMPM can then be expressed as:

$$
\widetilde{M}_{x, y}=\sum_{k=1}^{N_{1}} \sum_{l=1}^{N_{2}} \alpha_{k}^{x} \beta_{l \mid k}^{y} \widetilde{N}_{\alpha_{k}} \widetilde{N}_{\beta_{l \mid k}}, \quad x=0, \cdots, 2 N_{1}-2, y=0, \cdots, 2 N_{2}-2,
$$

where $N_{1}$ and $N_{2}$ are the maximum numbers of the particle sizes $\alpha_{k}$ and $\beta_{l \mid k}$, respectively. By construction, the particle size coordinates and weighted particle number generated in BVMPM should ensure that the corresponding moments are always equal to those from the true bivariate NDF:

$$
\widetilde{M}_{x, y}=M_{x, y}, \quad x=0, \cdots, 2 N_{1}-2, y=0, \cdots, 2 N_{2}-2 .
$$

With BVMPM, the moment evolution equation is transformed as: 
with the specific moment source terms given as:

$$
\begin{aligned}
& R_{x, y}(\widetilde{M})=K_{\mathrm{In}} i_{0}^{x} j_{0}^{y}, \\
& W_{x, y}(\widetilde{M})=K_{\mathrm{G}} \sum_{m=0}^{x} \sum_{n=0}^{y}\left(\begin{array}{l}
x \\
m
\end{array}\right)\left(\begin{array}{l}
y \\
n
\end{array}\right) \delta_{i}^{x-m} \delta_{j}^{y-n} \widetilde{M}_{m, n}-K_{\mathrm{G}} \widetilde{M}_{x, y}, \\
& S_{x, y}(\widetilde{M}, \widetilde{N})=K_{\mathrm{Sk}} \sum_{m=0}^{x} \sum_{n=0}^{y}\left(\begin{array}{l}
x \\
m
\end{array}\right)\left(\begin{array}{l}
y \\
n
\end{array}\right)\left(-\delta_{i}\right)^{x-m}\left(-\delta_{j}\right)^{y-n} \widetilde{M}_{m, n}-K_{\mathrm{Sk}} \widetilde{M}_{x, y} \\
& -K_{\mathrm{Sk}} \sum_{l=1}^{N_{2}}\left(\alpha_{1}-\delta_{i}\right)^{x}\left(\beta_{l \mid 1}-\delta_{j}\right)^{y} \widetilde{N}_{\alpha_{1}} \tilde{N}_{\beta_{l \mid 1}} \\
& -K_{\mathrm{Sk}} \sum_{k=1}^{N_{1}}\left(\alpha_{k}-\delta_{i}\right)^{x}\left(\beta_{1 \mid k}-\delta_{j}\right)^{y} \widetilde{N}_{\alpha_{k}} \widetilde{N}_{\beta_{1 \mid k}} \\
& +K_{\mathrm{Sk}}\left(\alpha_{1}-\delta_{i}\right)^{x}\left(\beta_{1 \mid 1}-\delta_{j}\right)^{y} \widetilde{N}_{\alpha_{1}} \widetilde{N}_{\beta_{1 \mid 1}} \text {, } \\
& G_{x, y}(\widetilde{M})=\frac{1}{2} K_{\mathrm{Cg}} \sum_{m=0}^{x} \sum_{n=0}^{y}\left(\begin{array}{l}
x \\
m
\end{array}\right)\left(\begin{array}{l}
y \\
n
\end{array}\right) \widetilde{M}_{m, n} \widetilde{M}_{x-m, y-n}-K_{\mathrm{Cg}} \widetilde{M}_{x, y} \widetilde{M}_{0,0}, \\
& F_{x, y}(\widetilde{M}, \widetilde{N})=K_{\mathrm{Fg}} \sum_{m=0}^{x} \sum_{n=0}^{y}\left(\begin{array}{l}
x \\
m
\end{array}\right)\left(\begin{array}{l}
y \\
n
\end{array}\right)\left(-i_{0}\right)^{x-m}\left(-j_{0}\right)^{y-n} \widetilde{M}_{m, n}+K_{\mathrm{Fg}} i_{0}^{x} j_{0}^{y} \widetilde{M}_{0,0}-K_{\mathrm{Fg}} \widetilde{M}_{x, y} \\
& -K_{\mathrm{Fg}} \sum_{l=1}^{N_{2}}\left(\left(\alpha_{1}-i_{0}\right)^{x}\left(\beta_{l \mid 1}-j_{0}\right)^{y}+i_{0}^{x} j_{0}^{y}-\alpha_{1}^{x} \beta_{l \mid 1}^{y}\right) \widetilde{N}_{\alpha_{1}} \widetilde{N}_{\beta_{l \mid 1}} \\
& -K_{\mathrm{Fg}} \sum_{k=1}^{N_{1}}\left(\left(\alpha_{k}-i_{0}\right)^{x}\left(\beta_{1 \mid k}-j_{0}\right)^{y}+i_{0}^{x} j_{0}^{y}-\alpha_{k}^{x} \beta_{1 \mid k}^{y}\right) \widetilde{N}_{\alpha_{k}} \widetilde{N}_{\beta_{1 \mid k}} \\
& +K_{\mathrm{Fg}}\left(\left(\alpha_{1}-i_{0}\right)^{x}\left(\beta_{1 \mid 1}-j_{0}\right)^{y}+i_{0}^{x} j_{0}^{y}-\alpha_{1}^{x} \beta_{1 \mid 1}^{y}\right) \widetilde{N}_{\alpha_{1}} \tilde{N}_{\beta_{1 \mid 1}} .
\end{aligned}
$$

${ }_{238}$ The challenge now is determining $\alpha_{k}, \beta_{l \mid k}, \widetilde{N}_{\alpha_{k}}$ and $\widetilde{N}_{\beta_{l \mid k}}$ such that Eq. (21) 239 is true while fulfilling the requirement that $\alpha_{1}=i_{0}$ and $\beta_{1 \mid k}=j_{0}$ to close the 

particles with the empirical marginal moments:

$$
\widetilde{M}_{x, 0}=\sum_{k=1}^{N_{1}} \alpha_{k}^{x} \widetilde{N}_{\alpha_{k}} \quad x=0, \cdots, 2 N_{1}-2 .
$$

243

$$
\widetilde{M}_{y \mid k}=\sum_{l=1}^{N_{2}} \beta_{l \mid k}^{y} \widetilde{N}_{\beta_{l \mid k}}, \quad y=0, \cdots, 2 N_{2}-2
$$

248 Firstly, rewrite Eq. (20) as a linear system:

$$
\mathbf{V R}=\mathbf{P}
$$

249 where

$$
\mathbf{V}=\left[\begin{array}{cccc}
\widetilde{N}_{\alpha_{1}} & \widetilde{N}_{\alpha_{2}} & \cdots & \widetilde{N}_{\alpha_{N_{1}}} \\
\alpha_{1} \widetilde{N}_{\alpha_{1}} & \alpha_{2} \widetilde{N}_{\alpha_{2}} & \cdots & \alpha_{N_{1}} \widetilde{N}_{\alpha_{N_{1}}} \\
\alpha_{1}^{2} \widetilde{N}_{\alpha_{1}} & \alpha_{2}^{2} \widetilde{N}_{\alpha_{2}} & \cdots & \alpha_{N_{1}}^{2} \widetilde{N}_{\alpha_{N_{1}}} \\
\vdots & \vdots & \vdots & \vdots \\
\alpha_{1}^{N_{1}-1} \widetilde{N}_{\alpha_{1}} & \alpha_{2}^{N_{1}-1} \widetilde{N}_{\alpha_{2}} & \cdots & \alpha_{N_{1}}^{N_{1}-1} \widetilde{N}_{\alpha_{N_{1}}}
\end{array}\right]
$$




$$
\mathbf{R}=\left[\begin{array}{cccc}
\widetilde{M}_{1 \mid 1} & \widetilde{M}_{2 \mid 1} & \cdots & \widetilde{M}_{2 N_{2}-2 \mid 1} \\
\widetilde{M}_{1 \mid 2} & \widetilde{M}_{2 \mid 2} & \cdots & \widetilde{M}_{2 N_{2}-2 \mid 2} \\
\widetilde{M}_{1 \mid 3} & \widetilde{M}_{2 \mid 3} & \cdots & \widetilde{M}_{2 N_{2}-2 \mid 3} \\
\vdots & \vdots & \vdots & \vdots \\
\widetilde{M}_{1 \mid N_{1}} & \widetilde{M}_{2 \mid N_{1}} & \cdots & \widetilde{M}_{2 N_{2}-2 \mid N_{1}}
\end{array}\right]
$$

250 and

$$
\mathbf{P}=\left[\begin{array}{cccc}
\widetilde{M}_{0,1} & \widetilde{M}_{0,2} & \cdots & \widetilde{M}_{0,2 N_{2}-2} \\
\widetilde{M}_{1,1} & \widetilde{M}_{1,2} & \cdots & \widetilde{M}_{1,2 N_{2}-2} \\
\widetilde{M}_{2,1} & \widetilde{M}_{2,2} & \cdots & \widetilde{M}_{2,2 N_{2}-2} \\
\vdots & \vdots & \vdots & \vdots \\
\widetilde{M}_{N_{1}-1,1} & \widetilde{M}_{N_{1}-1,2} & \cdots & \widetilde{M}_{N_{1}-1,2 N_{2}-2}
\end{array}\right]
$$

${ }_{251}$ Given the values for distinct $\alpha_{k}$ and non-negative $\widetilde{N}_{\alpha_{k}}$, the matrix $\mathbf{V}$ is nonsingular and the linear system in Eq. (30) can be solved by simply reversing the matrix $\mathbf{V}$ to determine the values for the conditional moments $\widetilde{M}_{y \mid k}$, which can then be adopted to find the conditional weighted particles by using the 1-D Blumstein-Wheeler algorithm.

The 2-step procedure illustrated above to find the bivariate weighted particles is described as a 2-D Blumstein-Wheeler algorithm presented in Appendix Appendix C. With the weighted particles determined, the moment source terms are closed. The numerical procedure of BVMPM is summarized in Algorithm 1. 
Algorithm 1: Bivariate Moment projection method algorithm.

Input: Moments of the NDF $M_{x, y}\left(t_{0}\right)$ for $x=0, \ldots, 2 N_{1}-2$ and $y=0, \ldots, 2 N_{2}-2$ or the NDF itself $N\left(t_{0} ; i, j\right)$ for $i=i_{0}, \ldots, \infty$ and $j=j_{0}, \ldots, \infty$ at initial time $t_{0}$; final time $t_{\mathrm{f}}$.

Output: Empirical moments of the NDF $\widetilde{M}_{x, y}\left(t_{\mathrm{f}}\right)$ for $x=0, \ldots, 2 N_{1}-2$ and $y=0, \ldots, 2 N_{2}-2$ at final time.

Calculate the moments of the true NDF using Eq. (9):

$$
M_{x, y}\left(t_{0}\right)=\sum_{i=i_{0}}^{\infty} \sum_{j=j_{0}}^{\infty} i^{x} j^{y} N(i, j)
$$

For $\widetilde{M}_{x, y}=M_{x, y}$, solve Eq. (20) for $\alpha_{k}$ and $\widetilde{N}_{\alpha_{k}}, \beta_{l}$ and $\widetilde{N}_{\beta_{l \mid k}}$ $\left(k=1, \ldots, N_{1}, l=1, \ldots, N_{2}\right)$ with $\alpha_{1}$ fixed at $i_{0}$ and $\beta_{1 \mid k}$ fixed at $j_{0}$ using the 2-D Blumstein and Wheeler algorithm:

$t \longleftarrow t_{0}, \widetilde{M}_{x, y}(t) \longleftarrow \widetilde{M}_{x, y}\left(t_{0}\right)$

while $t<t_{f}$ do

Integrate Eq. (22) over the time interval $\left[t_{i}, t_{i}+h\right]$ :

$$
\frac{\mathrm{d} \widetilde{M}_{x, y}}{\mathrm{~d} t}=R_{x, y}(\widetilde{M})+W_{x, y}(\widetilde{M})+S_{x, y}(\widetilde{M}, \widetilde{N})+G_{x, y}(\widetilde{M})+F_{x, y}(\widetilde{M}, \widetilde{N})
$$

where $R_{x, y}(\widetilde{M}), W_{x, y}(\widetilde{M}), S_{x, y}(\widetilde{M}, \widetilde{N}), G_{x, y}(\widetilde{M})$ and $F_{x, y}(\widetilde{M}, \widetilde{N})$ are given by Eqs. (23), (24), (25), (26) and (27) respectively.

Use the 2-D Blumstein algorithm to update $\alpha_{k}, \widetilde{N}_{\alpha_{k}}, \beta_{l \mid k}$ and $\widetilde{N}_{\beta_{l \mid k}}$, and assign solution at $t_{i+1}=t_{i}+h$ :

$$
\widetilde{M}_{x, y}\left(t_{i+1}\right) \leftarrow \widetilde{M}_{x, y}\left(t_{i}+h\right)
$$

$i \longleftarrow i+1$ 


\section{Results and discussion}

In this section, the performance of BVMPM for solving the bivariate PBEs is assessed. The method is first tested for the individual particle processes of inception, growth, shrinkage, coagulation and fragmentation, then for all of these processes combined. We devise a number of test cases where different types of NDFs are supplied as the initial conditions. The numerical results are compared to those from HMOM and a high-precision stochastic solution calculated using the direct simulation algorithm (DSA).

\subsection{Inception}

As mentioned above, inception is modeled as the formation of the smallest particles. In this work, the inception rate is assumed to be a constant: $K_{\text {In }}=10^{12} \mathrm{~s}^{-1}$. Simulations are performed with a normal distribution as the initial condition:

$$
N(i, j)=100 \exp \left(-1\left((i-100)^{2}+(j-100)^{2}\right) / 200\right),
$$

which is shown in Fig. 1. Also shown in Fig. 1 is the NDF computed by solving the master equation after 100 seconds of pure inception. Only the smallest particles at $\left(i_{0}, j_{0}\right)$ are formed while the number of the other particles remains unchanged.

We now want to see if BVMPM is able to capture this increase in the number of the smallest particles due to inception. We use in total $16\left(N_{1}=\right.$ $\left.4, N_{2}=4\right)$ weighted particle size coordinates to simulate this process. Figure 2 exhibits the distributions of these weighted particles at $t_{0}$ and $t_{\mathrm{f}}$. At $t_{0}$, most of the weighted particles are located at around $(100,100)$. Some 

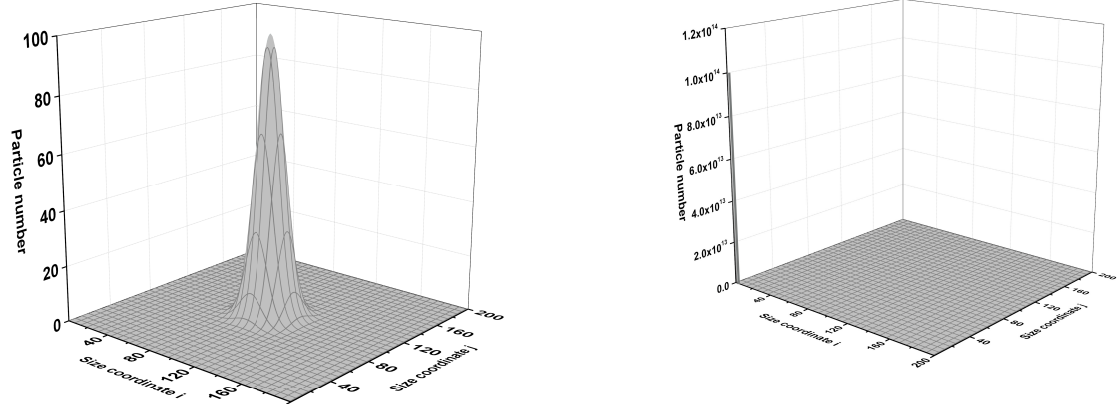

Figure 1: Particle number density functions at $t_{0}=0 \mathrm{~s}$ (left panel) and $t_{f}=100 \mathrm{~s}$ (right panel) computed by solving the master equation under pure inception.

weighted particles are observed to be located at the smallest size coordinates, suggesting that the proposed 2-D Blumstein and Wheeler algorithm successfully fixes the weighted particles at the designated location. A significant increase in the number of the weighted particles at $\left(i_{0}, j_{0}\right)$ is observed at the end of simulation, this trend matches well to the observation in Fig.1.
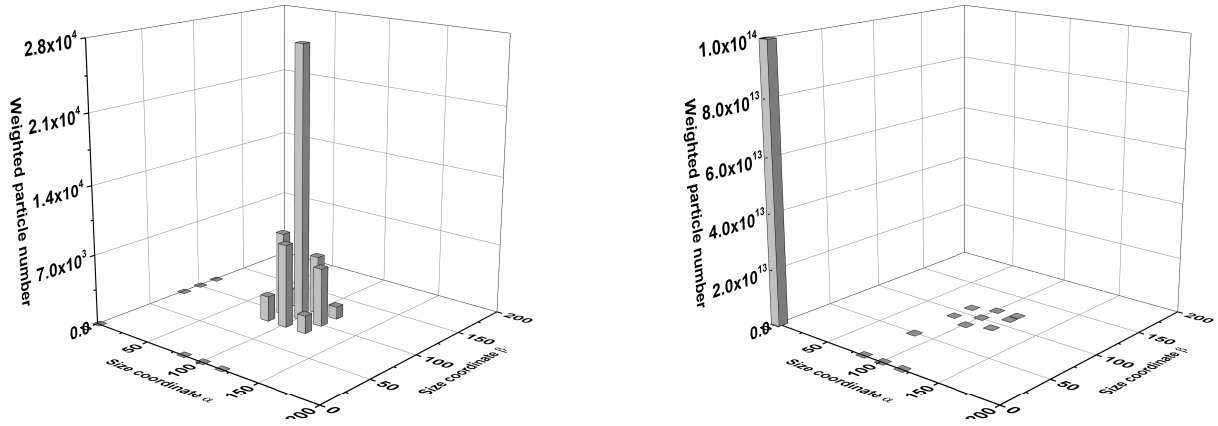

Figure 2: Distributions of weighted particles at $t_{0}=0 \mathrm{~s}$ (left panel) and $t_{f}=100 \mathrm{~s}$ (right panel) generated in BVMPM under pure inception.

As a further point of comparison, the time evolutions of $M_{0,0}, M_{0,1}, M_{1,0}$ and $M_{1,1}$ computed using BVMPM, HMOM and the stochastic method are 

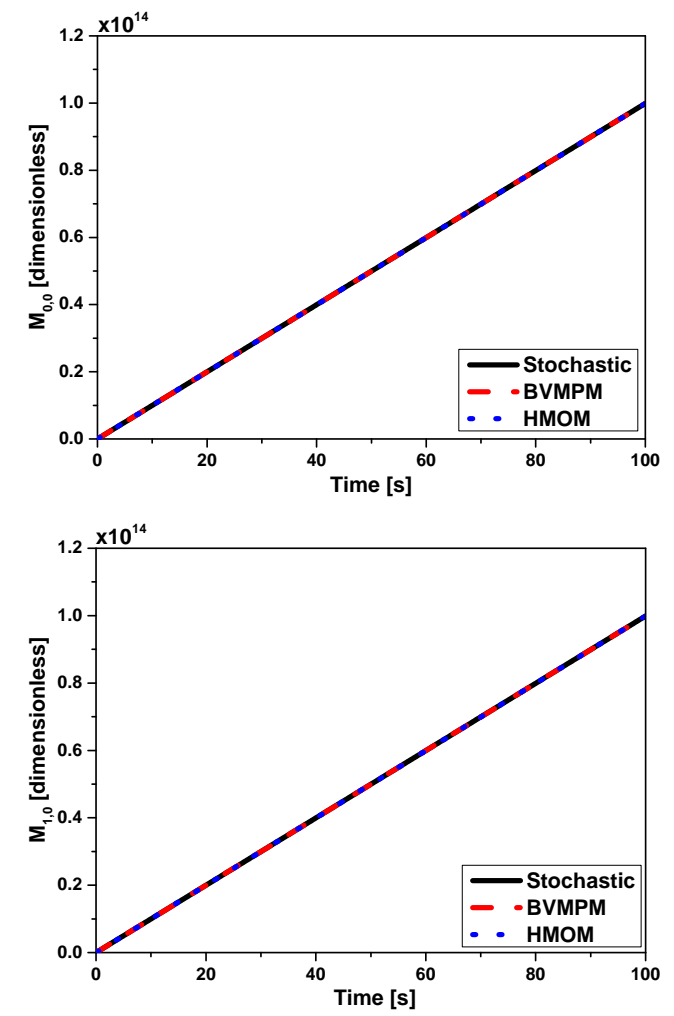
number and sizes of particles.
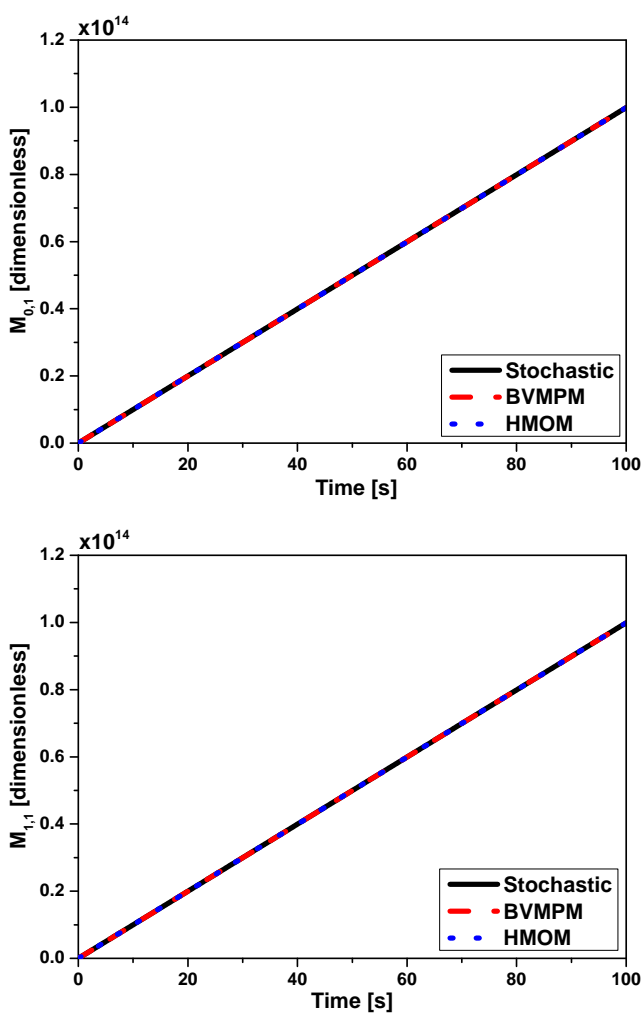

Figure 3: Comparison of $M_{0,0}$ (top left panel), $M_{0,1}$ (top right panel), $M_{1,0}$ (bottom left panel) and $M_{1,1}$ (bottom right panel) between BVMPM, HMOM and the stochastic method under pure inception.

shown in Fig. 3. It can be seen that all the methods give the same results. The continuous inception of particles leads to a linear increase in the total 
that any positive value can be taken as the size change and it can be different for both size coordinates. A constant growth kernel is adopted: $K_{\mathrm{G}}=2 \mathrm{~s}^{-1}$, and the following uniform distribution is applied as the initial condition:

$$
N(i, j)=1, \quad i=1,2, \cdots, 20, j=1,2, \cdots, 20 .
$$

The NDF at $t_{0}$ and that at $t_{\mathrm{f}}$ computed by solving the master equation after 50 seconds for pure growth are shown in Fig. 4. A shift of particles towards the larger size coordinates is observed; however, the distribution becomes widened and the peak decreases in magnitude consistent with a growth process.
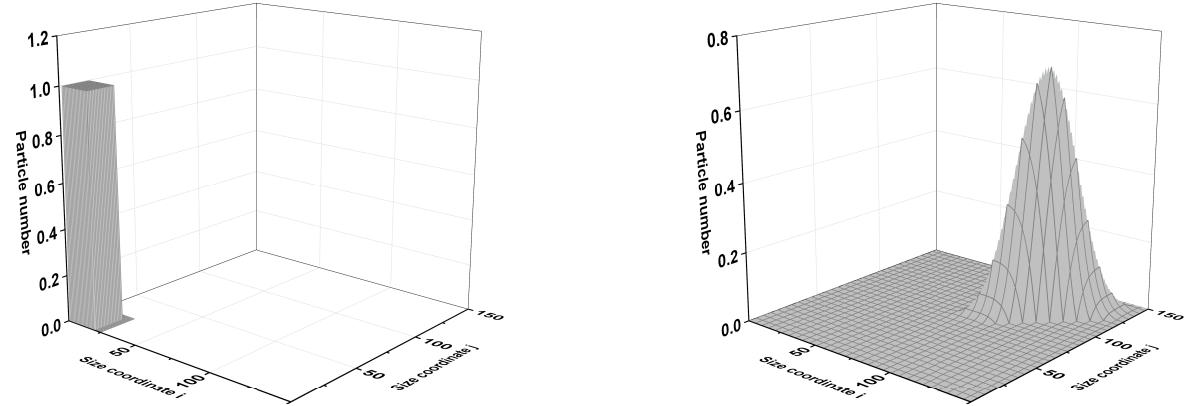

Figure 4: Particle number density functions at $t_{0}=0 \mathrm{~s}$ (left panel) and $t_{f}=50 \mathrm{~s}$ (right panel) computed by solving the master equation under pure growth.

Figure 5 shows the distributions of the weighted particles generated in BVMPM to approximate the NDFs at $t_{0}$ and $t_{\mathrm{f}}$. Similar to Fig. 4 , the weighted particles have shifted towards the larger size coordinates reflecting the increase in the particle sizes.

The time evolution of $M_{0,0}, M_{0,1}, M_{1,0}$ and $M_{1,1}$ computed using the different methods are compared in Fig. 6. Since constant kernels are used, 

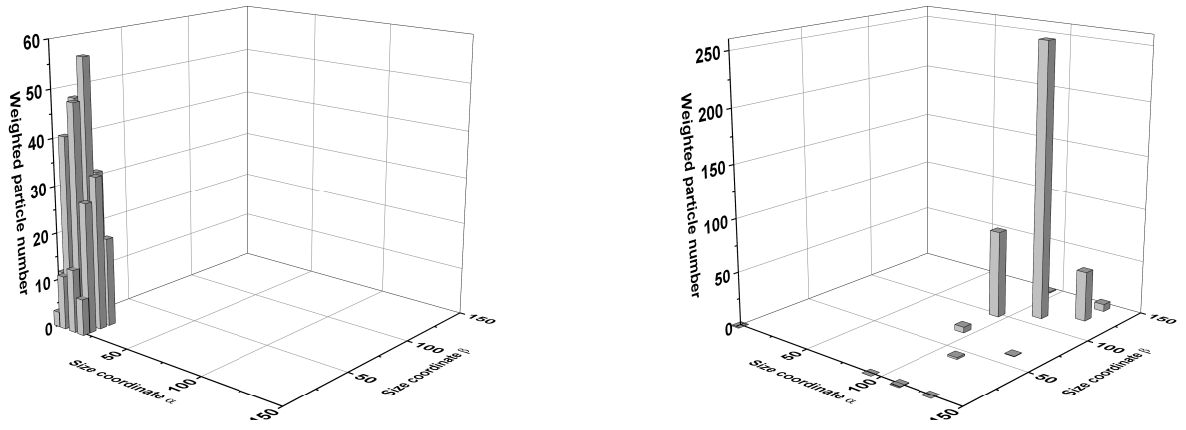

Figure 5: Distributions of weighted particles at $t_{0}=0 \mathrm{~s}$ (left panel) and $t_{f}=50 \mathrm{~s}$ (right panel) generated in BVMPM under pure growth.

no fractional- or negative-order moments are present in the moment source term. Both HMOM and BVMPM give the same results with the stochastic method. The total particle number reflected by $M_{0,0}$ remains unchanged, while a linear increase is observed for the particle sizes indicated by $M_{0,1}$ and $M_{1,0}$.

\subsection{Coagulation}

Coagulation is a nonlinear process describing the collision and sticking among particles. In this work, the coagulation kernel is assumed to be $K_{\mathrm{Cg}}=$ $1 \times 10^{-6} \mathrm{~s}^{-1}$. A log-normal distribution is adopted as the initial condition:

$$
N(i, j)=100 \exp \left(-\left((\log (i)-\log (50))^{2}+(\log (j)-\log (50))^{2}\right) / 2\right) .
$$

The NDFs at the beginning and end of the simulation are shown in Fig. 7. A shift of the distribution towards the larger particle sizes is observed as particles collide and stick together. 

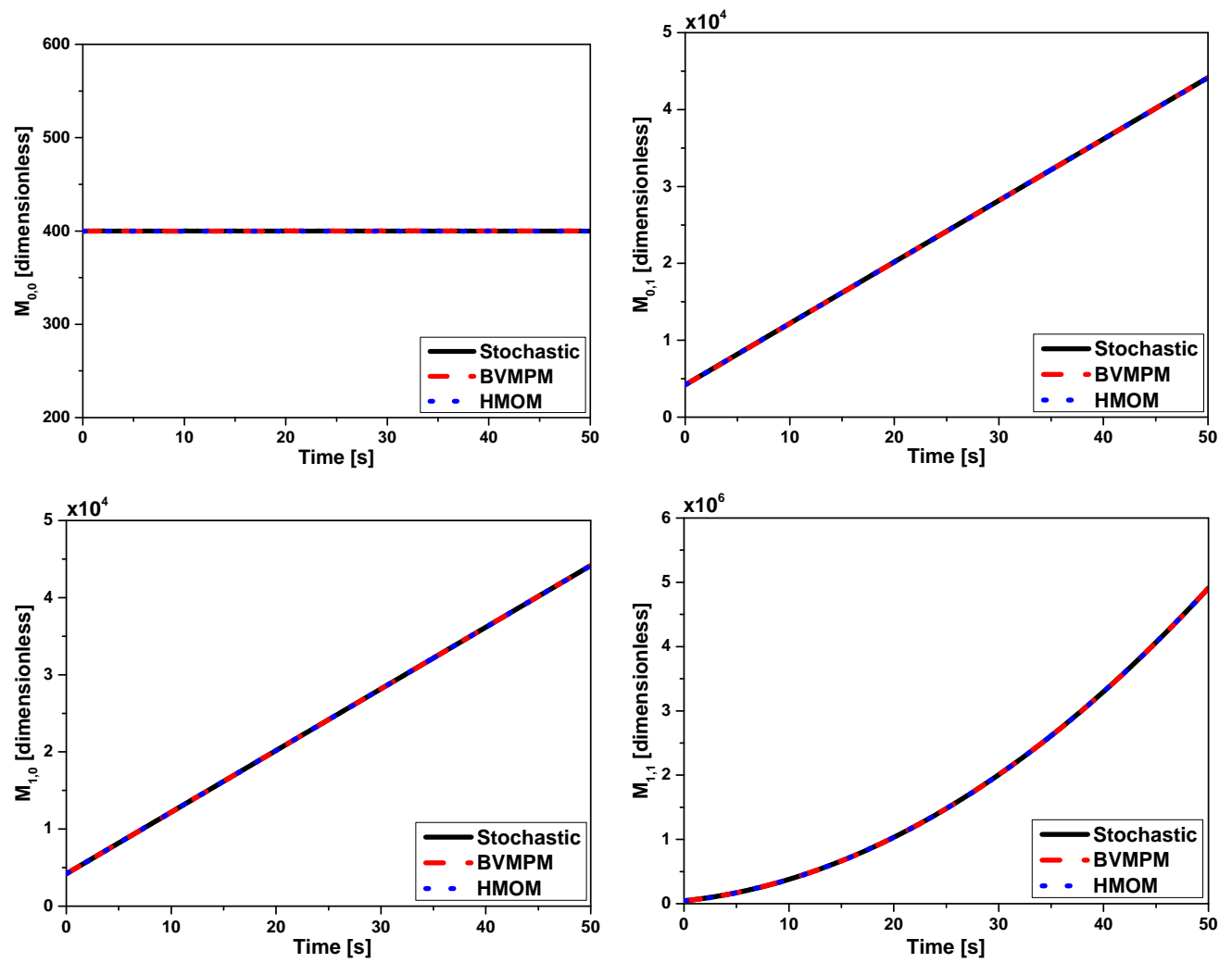

Figure 6: Comparison of $M_{0,0}$ (top left panel), $M_{0,1}$ (top right panel), $M_{1,0}$ (bottom left panel) and $M_{1,1}$ (bottom right panel) between BVMPM, HMOM and the stochastic method under pure growth.

Figure 8 shows the formation of weighted particles at large size coordinates together with the decrease of weighted particles at small size coordinates. This is consistent with the trend observed in Fig. 7.

The mean quantities computed using BVMPM are in agreement with HMOM and the stochastic method as shown in Fig. 9. Since coagulation is a nonlinear process, we observe a nonlinear decrease in $M_{0,0}$ while $M_{0,1}$ and $M_{1,0}$ remain unchanged. 

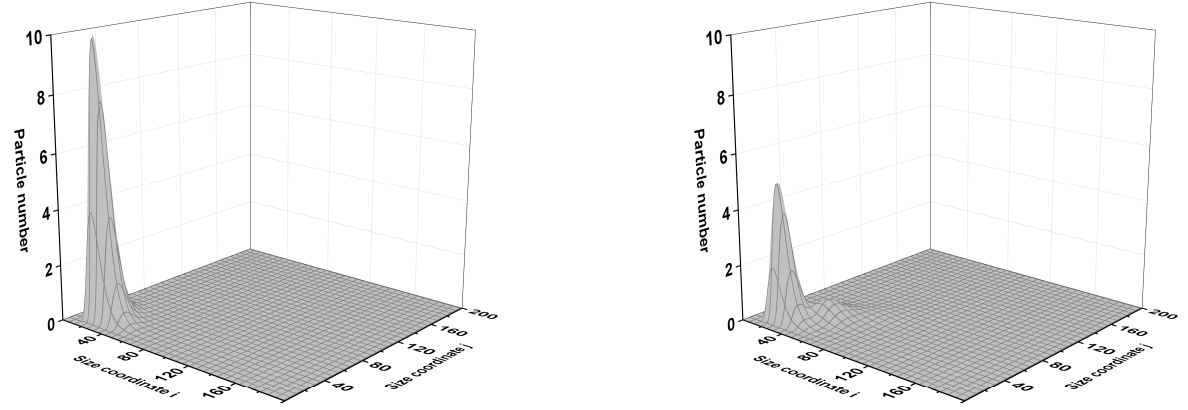

Figure 7: Particle number density functions at $t_{0}=0 \mathrm{~s}$ (left panel) and $t_{f}=30 \mathrm{~s}$ (right panel) obtained by the stochastic method for pure coagulation.
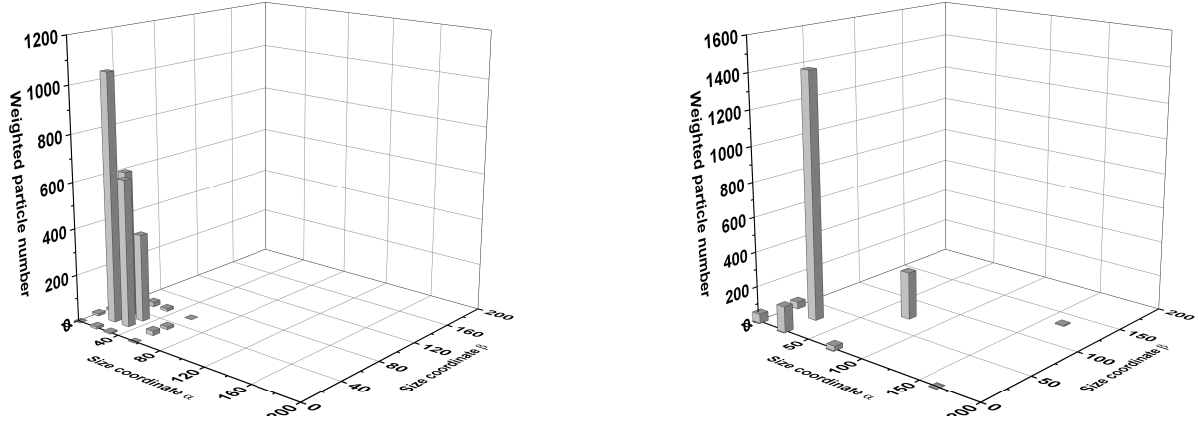

Figure 8: Distributions of weighted particles at $t_{0}=0 \mathrm{~s}$ (left panel) and $t_{f}=30 \mathrm{~s}$ (right panel) generated in BVMPM under pure coagulation.

\subsection{Shrinkage}

Shrinkage is the opposite of the growth process but with an important difference: when particles of the smallest sizes shrink they are removed from the particle system, leading to a decrease in the total particle number. As shown in Eq. (13), the number of particles of the smallest sizes is required to close the shrinkage moment source term. In BVMPM, we fix some particle sizes at the samllest size coordinates so that the cooresponding number of these weighted particles can be used to evaluate the boundary flux term due 

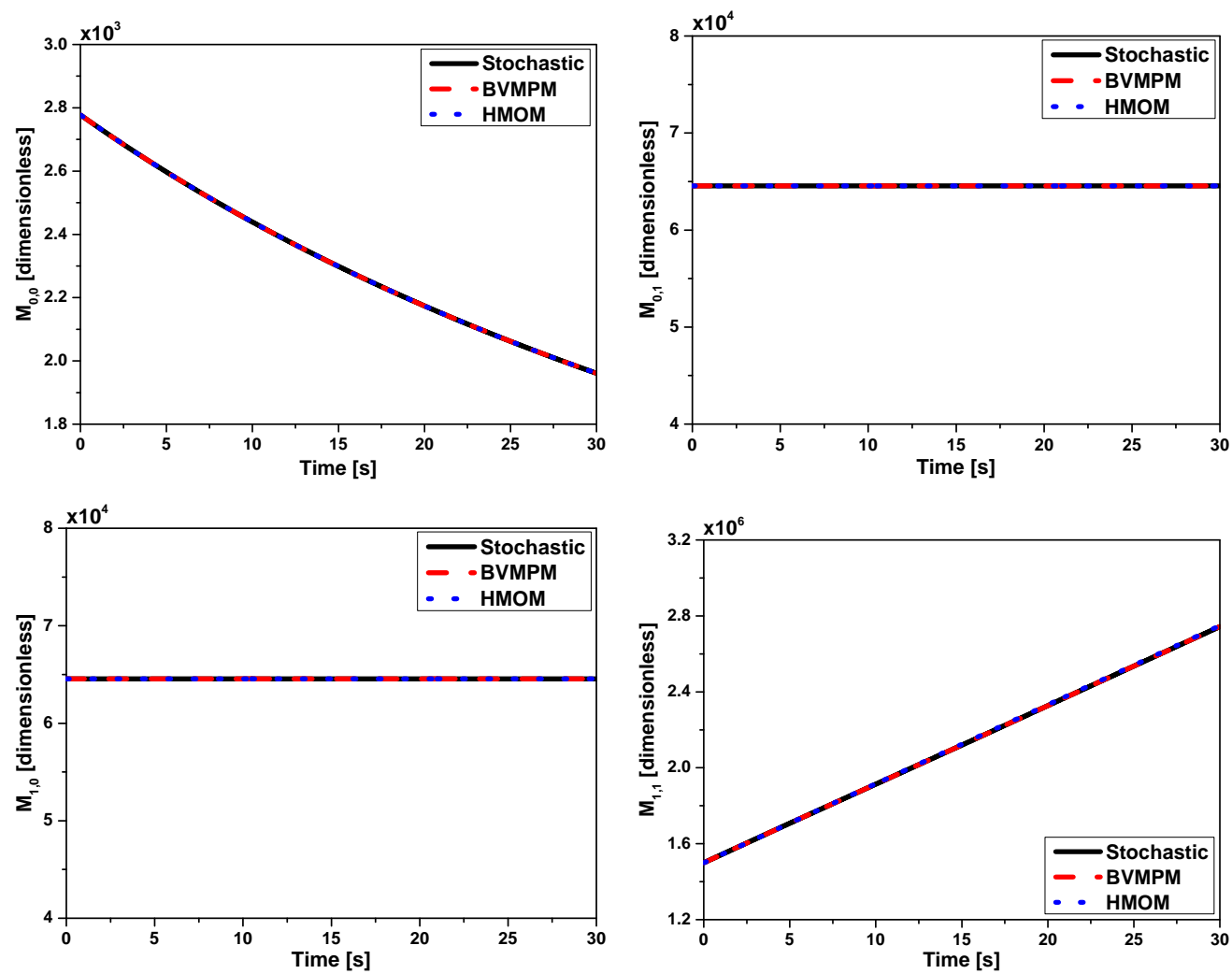

Figure 9: Comparison of $M_{0,0}$ (top left panel), $M_{0,1}$ (top right panel), $M_{1,0}$ (bottom left panel) and $M_{1,1}$ (bottom right panel) between BVMPM, HMOM and the stochastic method under pure coagulation.

to shrinkage. In this section, we test the ability of BVMPM to handle the shrinkage problem. A constant shrinkage kernel is used: $K_{\mathrm{sk}}=2 \mathrm{~s}^{-1}$ and the size change in one shrink event is assumed to be 1 . Two test cases are adopted where different types of NDFs are supplied as the initial condition.

Case 1 A normal distribution:

$$
N(i, j)=10^{20} \exp \left(-\left((i-100)^{2}+(j-100)^{2}\right) / 1000\right)
$$




$$
N(i, j)=10^{20} \exp \left(-\left((\log (i)-\log (100))^{2}+(\log (j)-\log (100))^{2}\right) / 0.02\right)
$$

For Case 1, a normal distribution is supplied as the initial condition which is shown in Fig. 10. Also shown in Fig. 10 is the NDF obtained by solving the master equation after 100 seconds of pure shrinkage. The NDF shifts towards the smallest particle size. A decrease in the total particle number is observed as the smallest particles are continuously removed from the particle system due to shrinkage.
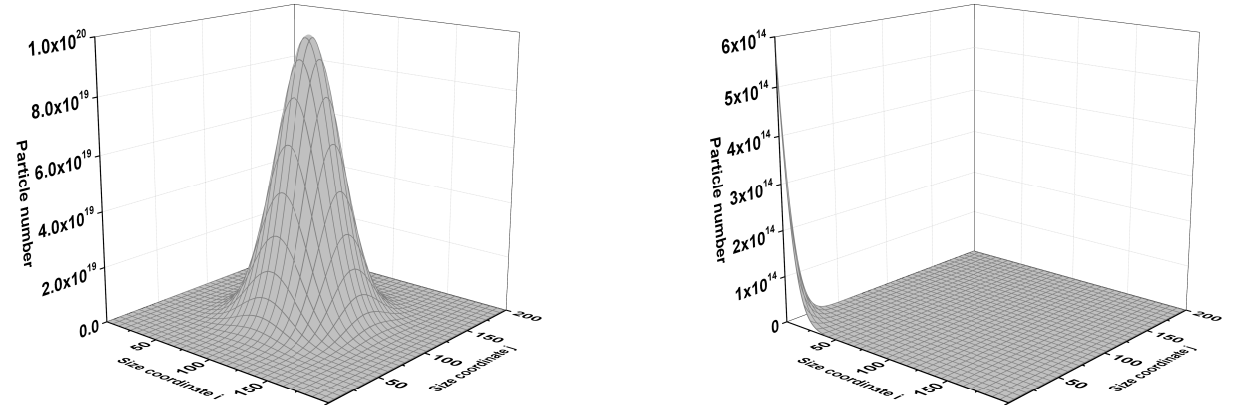

Figure 10: Particle number density functions at $t_{0}=0 \mathrm{~s}$ (left panel) and $t_{f}=100 \mathrm{~s}$ (right panel) computed by solving the master equation under pure shrinkage (Case 1).

The distributions of the weighted particles obtained in BVMPM $\left(N_{1}=\right.$ $\left.4, N_{2}=4\right)$ to approximate the NDFs are shown in Fig. 11. All the weighted particles are moving towards the smallest particle sizes. An increase in $\widetilde{N}_{1,1}$ is observed as the large particles are transformed into the smallest ones. This observation is consistent with that in Fig. 10. 

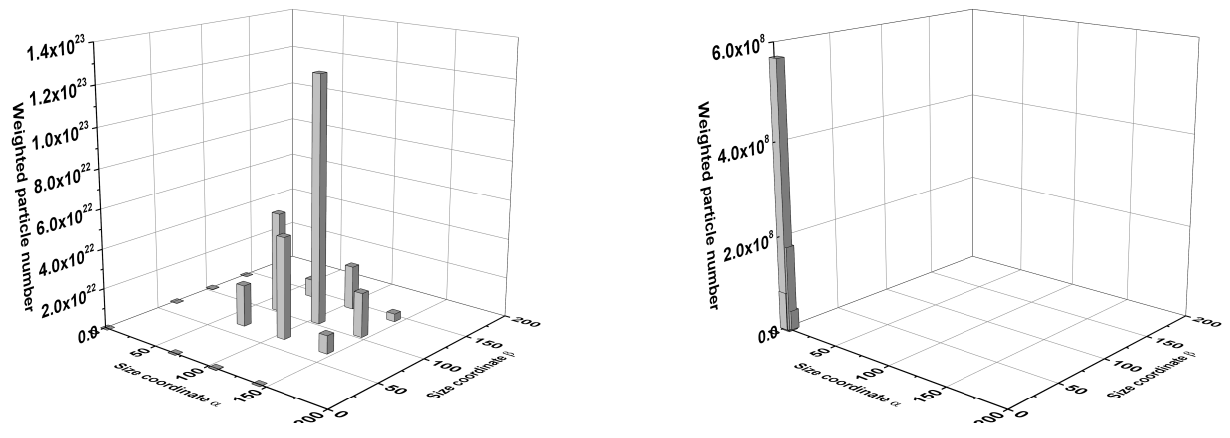

Figure 11: Distributions of weighted particles at $t_{0}=0 \mathrm{~s}$ (left panel) and $t_{f}=100 \mathrm{~s}$ (right panel) generated in BVMPM under pure shrinkage (Case 1).

To investigate the influence of the number of the weighted particle sizes on the accuracy of BVMPM, we vary $N_{2}$ from 3 to 5 while keeping $N_{1}$ unchanged. Note that the accuracy of BVMPM can also be affected by changing $N_{1}$ in a similar way. The $M_{0,0}, M_{0,1}, M_{1,0}$ and $M_{1,1}$ obtained using BVMPM for different $N_{2}$ are compared with the stochastic solution in Fig. 12. $M_{0,0}$ computed using BVMPM with $N_{2}=3$ (dashed line) shows an obvious discrepancy with $M_{0,0}$ obtained by the stochastic method (continuous line). By contrast, the moments obtained using $N_{2}=4$ and $N_{2}=5$ show a satisfactory agreement with the stochastic solution. This indicates that increasing the number of particle sizes in BVMPM can lead to a better approximation of the number of the smallest particles. Similar observations are found for $M_{0,1}$ and $M_{1,0}$. By contrast, $M_{1,1}$ is relatively insensitive to the number of particle sizes. $M_{1,1}$ obtained using BVMPM with $N_{2}=3,4$ and 5 all match well with the stochastic solution. Note that increasing the number of particle sizes requires the solution of more moments. Smaller tolerances have to be adopted for the time integration of the ODEs and the stiffness of the 

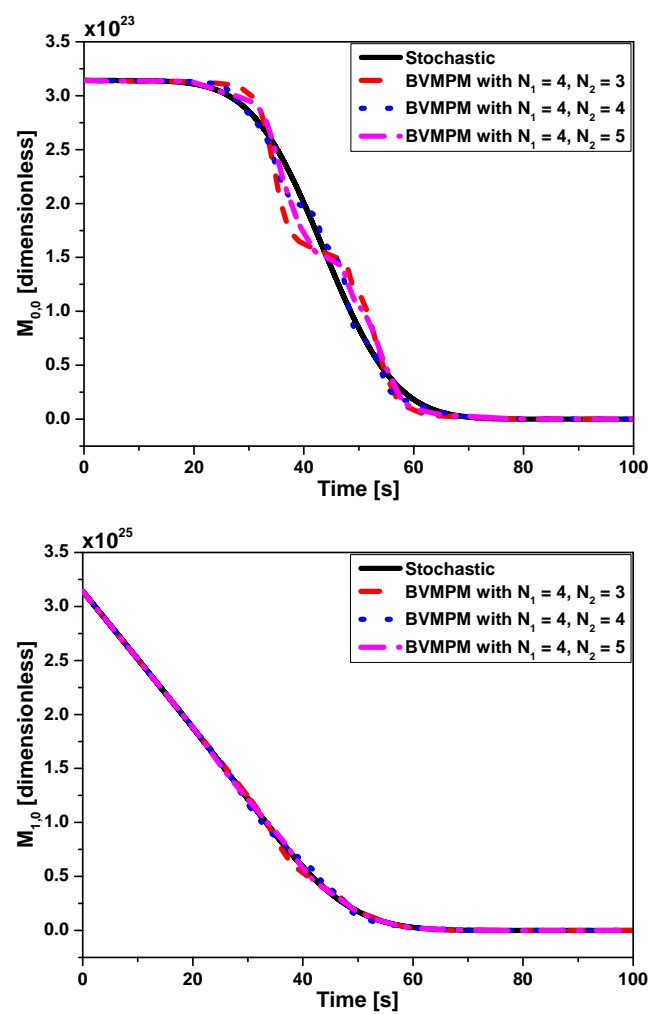

eigenvalue-eigenvector problem in the Blumstein and Wheeler algorithm is increased, resulting in a higher computational cost. For this reason, $N_{2}=4$ is a good compromise between accuracy and computational efficiency.
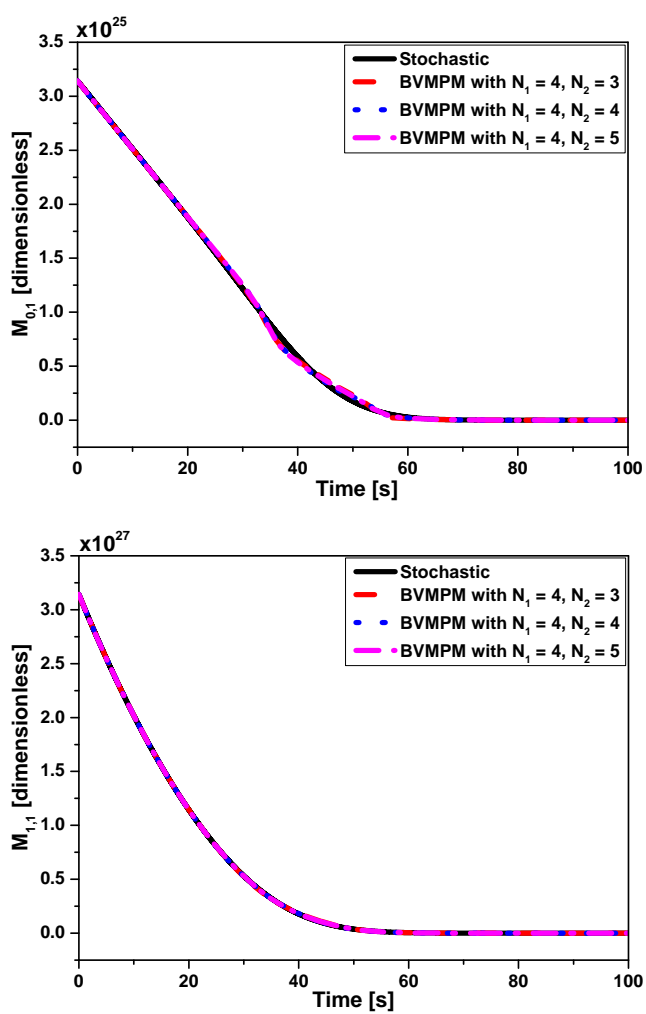

Figure 12: Sensitivity of $M_{0,0}$ (top left panel), $M_{0,1}$ (top right panel), $M_{1,0}$ (bottom left panel) and $M_{1,1}$ (bottom right panel) to the number of particle sizes, $N_{2}$, using BVMPM under pure shrinkage. Results coorspond to Case 1 where a normal distribution is supplied as the initial condition. The stochastic solution is shown as a point of reference.

Figure 13 compares the moments obtained using BVMPM, HMOM and the stochastic method. As mentioned above, In HMOM the NDF is discretized into a group of the smallest particles and a group of large particles. 
A source term accounting for the formation and consumption of the smallest particles is proposed. It is assumed that the number of the smallest particles formed due to the shrinkage of the large particles is proportional to the totoal sizes decreased from these large particles. This assumption is too coarse as there are cases where the NDF is located far away from the smallest sizes, for which the shrinkage process can lead to a decrease of the total particle size without there being a change in the total number of particles. As a result HMOM overestimates the formation of the smallest particles, and therefore $M_{0,0}$, at the beginning. Since the smallest particles are easier to remove, HMOM leads to a faster decrease in $M_{0,0}$ and, eventually underestimates the particle number $M_{0,0}$ and particle sizes $\left(M_{0,1}\right.$ and $\left.M_{1,0}\right)$. By contrast, the moments obtained uisng BVMPM with $N_{1}=4$ and $N_{2}=4$ match satisfactorily well to the stochastic solutions.

The results for Case 2 where a log-normal distribution is supplied as the initial condition are shown in Fig. 14. Similar to Case 1, in Case 2 HMOM overestimates the total particle number at the initial stage while the reverse occurs at the later stage. By contrast, BVMPM exhibits very high accuracy. Excellent agreement is achieved between the moments obtained using BVMPM and the stochastic method.

\subsection{Fragmentation}

Fragmentation is a popular phenomenon in particle dynamics. It is a process by which particles break up into two or more fragments, leading to an accumulation of particles at the smallest sizes. As a result, the information on the number of the smallest particles plays an important role. In this section, we test the performance of BVMPM in treating the fragmentation 

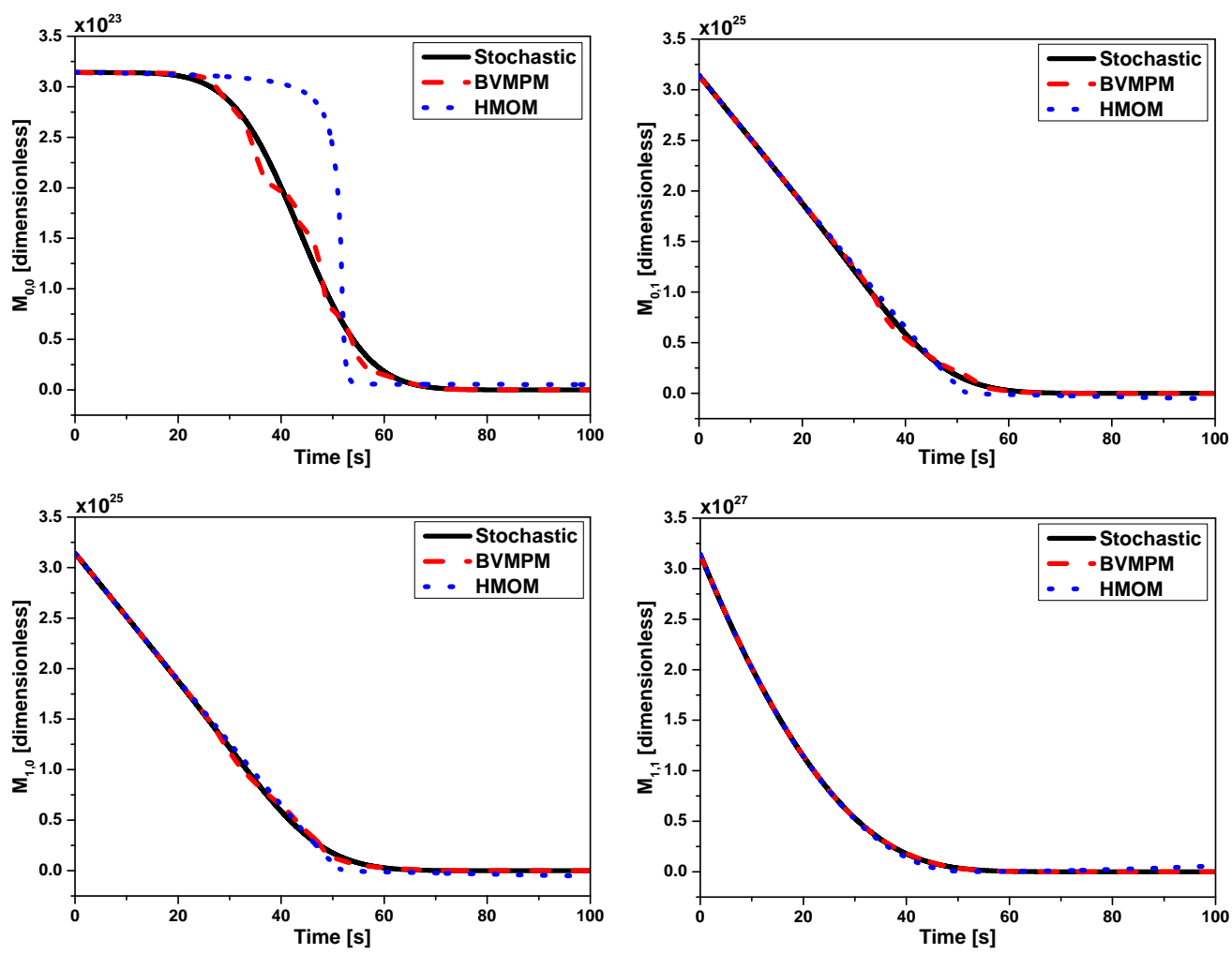

Figure 13: Comparison of $M_{0,0}$ (top left panel), $M_{0,1}$ (top right panel), $M_{1,0}$ (bottom left panel) and $M_{1,1}$ (bottom right panel) between BVMPM, HMOM and the stochastic method under pure shrinkage (Case 1).

process. The fragmentation kernel is assumed to be $K_{\mathrm{Fg}}=5 \mathrm{~s}^{-1}$. Two types of NDFs are supplied as the initial condition:

Case 3 A log-normal distribution:

$$
N(i, j)=10^{20} \exp \left(-\left((\log (i)-\log (100))^{2}+(\log (j)-\log (100))^{2}\right) / 0.2\right)
$$

Case 4 A uniform distribution: 

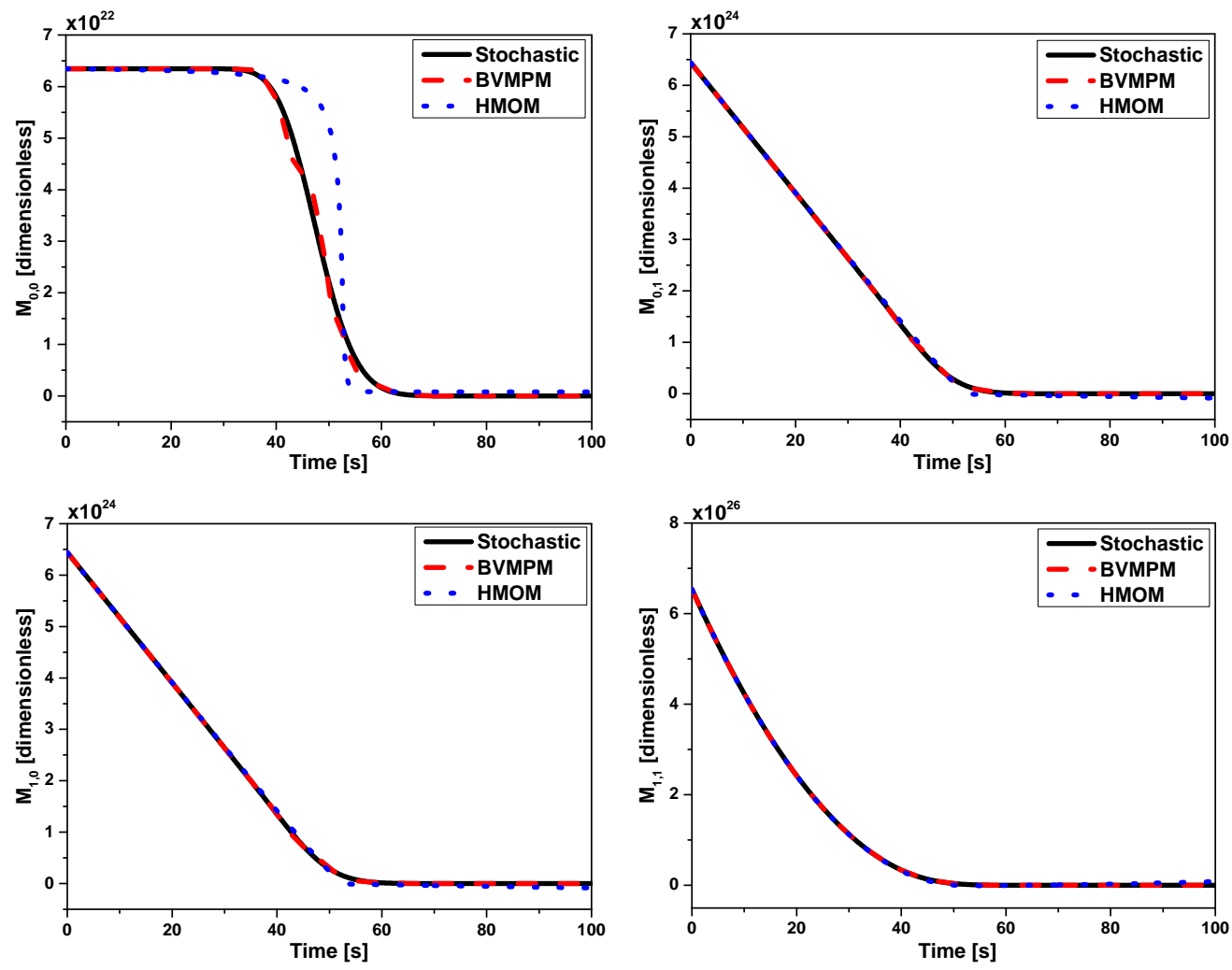

Figure 14: Comparison of $M_{0,0}$ (top left panel), $M_{0,1}$ (top right panel), $M_{1,0}$ (bottom left panel) and $M_{1,1}$ (bottom right panel) between BVMPM, HMOM and the stochastic method under pure shrinkage (Case 2).

$$
N(i, j)=100, \quad i=100, \cdots, 200, j=100, \cdots, 200 .
$$

For Case 3 a log-normal distribution is adopted as the initial condition as shown in Fig. 15. Also shown in Fig. 15 is the NDF obtained by solving the fragmentation master equation after 50 seconds. It can be seen that all particles have been transformed into the smallest ones.

Figure 16 shows the distributions of the weighted particles generated in 

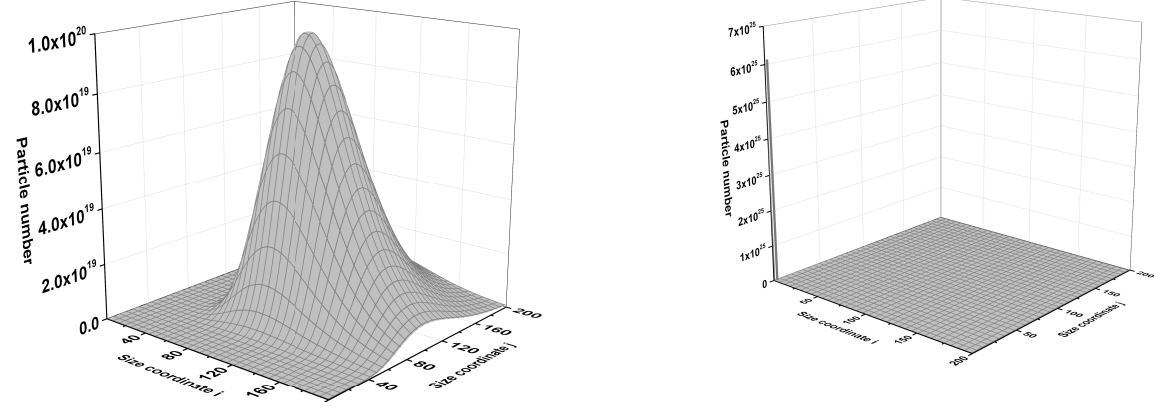

Figure 15: Particle number density functions at $t_{0}=0 \mathrm{~s}$ (left panel) and $t_{f}=50 \mathrm{~s}$ (right panel) computed by solving the master equation under pure fragmentation (Case 3).

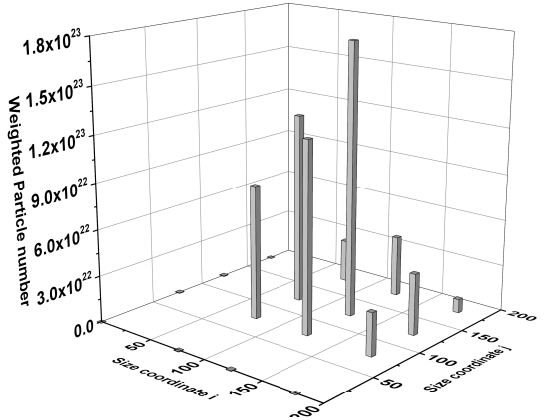
at $\left(i_{0}, j_{0}\right)$ is observed.

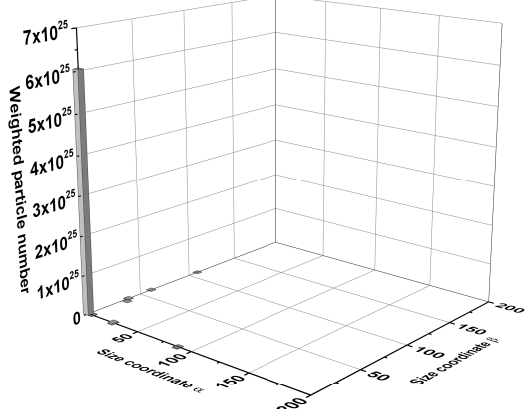

Figure 16: Distributions of weighted particles at $t_{0}=0 \mathrm{~s}$ (left panel) and $t_{f}=50 \mathrm{~s}$ (right panel) generated in BVMPM under pure fragmentation (Case 3).

BVMPM to simulate the fragmentation process. All the weighted particles shift towards the smallest particle size. An accumulation of weighted particles

Figure 17 compares the moments obtained using HMOM, BVMPM and the stochastic method. In general, BVMPM gives the same results with the stochastic solutions. The total number of particles represented by $M_{0,0}$ 
exhibits an increase at the beginning as the large particle breaks up into two smaller ones. Eventually $M_{0,0}$ reaches steady when all the particles have been transformed into the smallest ones which are not supposed to fragment any further. The total particle sizes $\left(M_{0,1}\right.$ and $\left.M_{1,0}\right)$ remain unchanged during the fragmentation process. As mentioned above, HMOM tends to overestimate the formation of the smallest particles due to the coarse assumption made on the smallest particle source terms. As a result, a higher $M_{0,0}$ is predicted by HMOM.

In Case 4, a uniform distribution is used as the initial condition. The moments obtained using different methods are compared in Fig. 18. The conclusions can be drawn are similar to that in Case 3: HMOM over-predicts the total number of particles; BVMPM exhibits very high accuracy, giving the same results with the stochastic method.

\subsection{Combined processes}

We have evaluated the ability of BVMPM to treat the individual particle processes of inception, coagulation, growth, shrinkage and fragmentation. Now we want to test BVMPM against HMOM and the stochastic method for all of these particle processes combined. The initial condition is defined as a log-normal distribution:

$$
N(i, j)=10^{10} \exp \left(-\left((\log (i)-\log (100))^{2}+(\log (j)-\log (100))^{2}\right) / 0.02\right)
$$

The kernels adopted are: $K_{\mathrm{In}}=10^{8} \mathrm{~s}^{-1}, K_{\mathrm{G}}=2 \mathrm{~s}^{-1}, K_{\mathrm{Cg}}=10^{-12} \mathrm{~s}^{-1}$, $K_{\mathrm{Sk}}=20 \mathrm{~s}^{-1}$ and $K_{\mathrm{Fg}}=10^{-4} \mathrm{~s}^{-1}$. Since the focus of this work is to test the ability of BVMPM to handle shrinkage, a larger shrinkage kernel than 

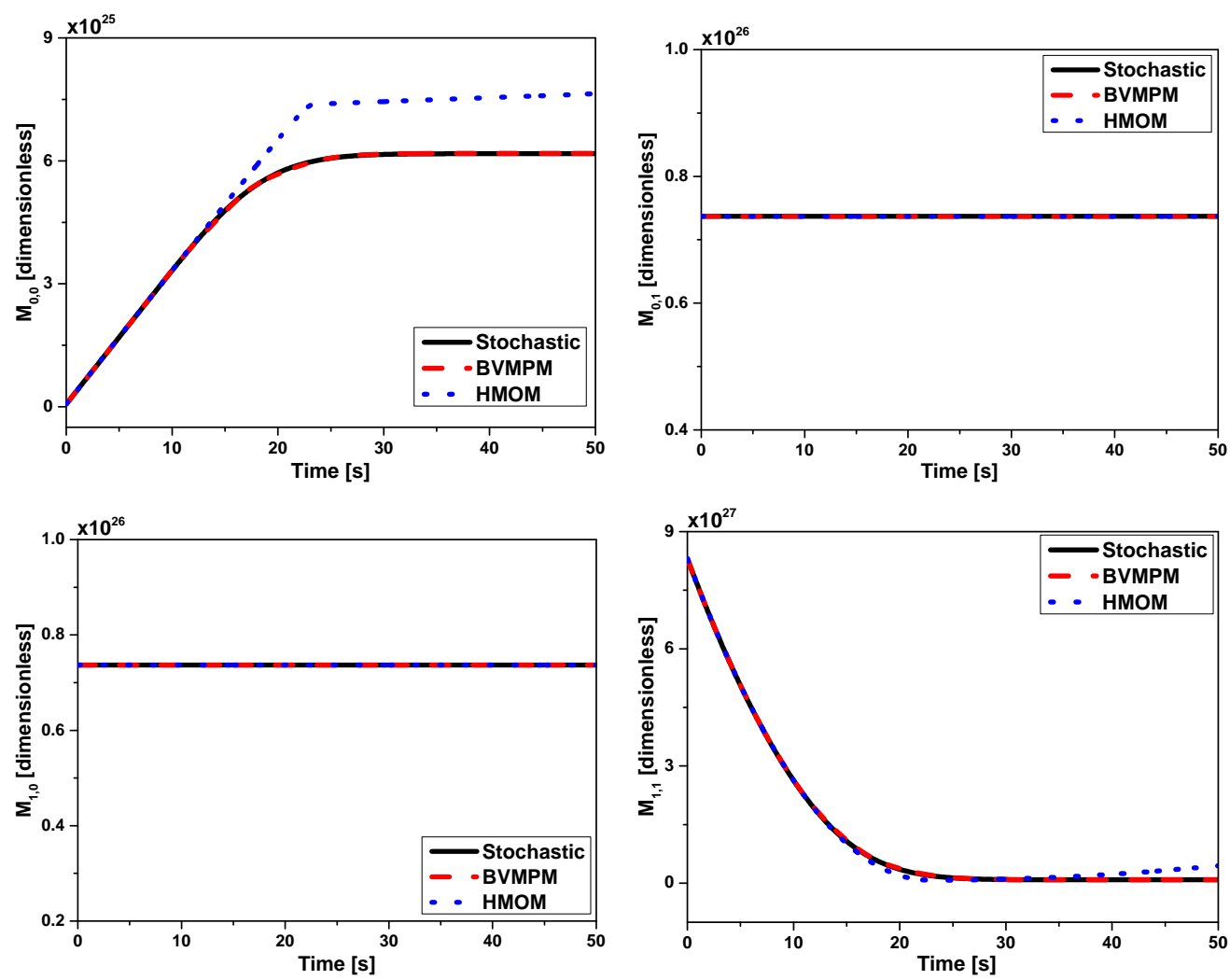

Figure 17: Comparison of $M_{0,0}$ (top left panel), $M_{0,1}$ (top right panel), $M_{1,0}$ (bottom left panel) and $M_{1,1}$ (bottom right panel) between BVMPM, HMOM and the stochastic method under pure fragmentation (Case 3).

the growth kernel is adopted to simulate a shrinkage dominate process. The NDFs at the beinning and end of the simulation under the combined processes are shown in Fig. 19. Figure 20 shows the evolution of the weighted particles for this case. There is a net shrinkage of particles and the NDF moves towards the smallest size coordinates.

Comparison of the moments between different methods is shown in Fig. 21. In general, the moments obtained by BVMPM match satisfactorily well to 

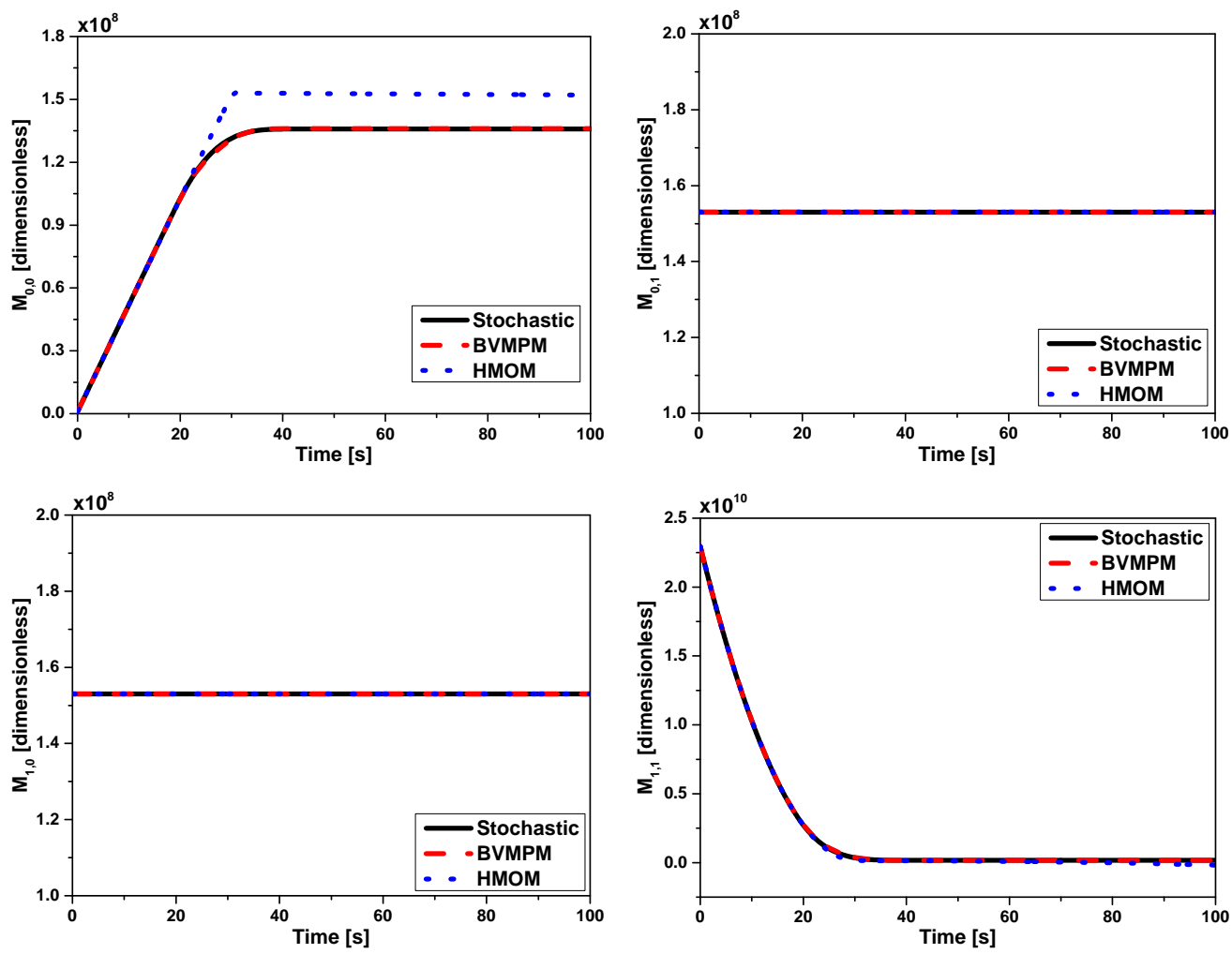

Figure 18: Comparison of $M_{0,0}$ (top left panel), $M_{0,1}$ (top right panel), $M_{1,0}$ (bottom left panel) and $M_{1,1}$ (bottom right panel) between BVMPM, HMOM and the stochastic method under pure shrinkage (Case 4).

447

the stochastic solutions. The total number of particles remains unchanged before $4 \mathrm{~s}$ since no particles exist at the smallest size coordinates. Then $M_{0,0}$ exhibits a fast decrease before reaching relatively steady. The moments obtained by HMOM show an obvious discrepency with the stochastic solutions due to the poor prediction on the number of the smallest particles. 

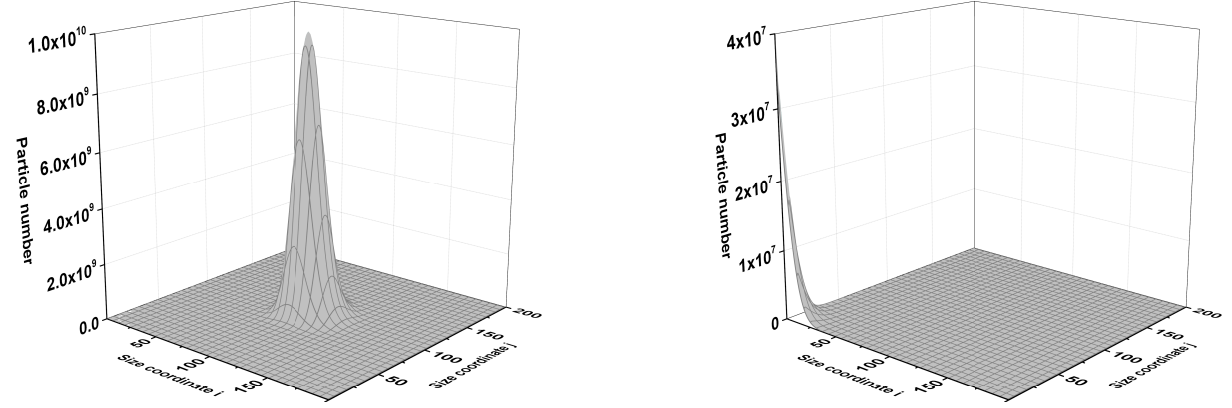

Figure 19: Particle number density functions at $t_{0}=0 \mathrm{~s}$ (left panel) and $t_{f}=8 \mathrm{~s}$ (right panel) computed using the stochastic method under all particle processes.
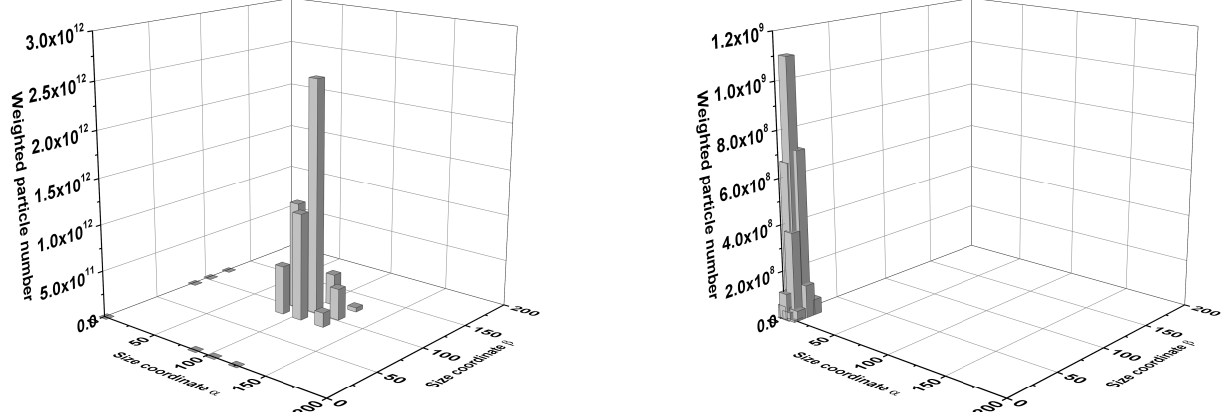

Figure 20: Distributions of weighted particles at $t_{0}=0 \mathrm{~s}$ (left panel) and $t_{f}=8 \mathrm{~s}$ (right panel) generated in BVMPM for all particle processes.

\section{Conclusion}

In this work, a bivariate moment projection method is proposed for solving the two-dimensional population balance equations describing particle dynamics. The general idea of this method is to consider the particle number density function (NDF) as a product of univariate marginal NDF and a conditional NDF. A 2-D Blumstein and Wheeler algorithm is introduced to approximate the NDF with a set of weighted particles. The sizes of some 

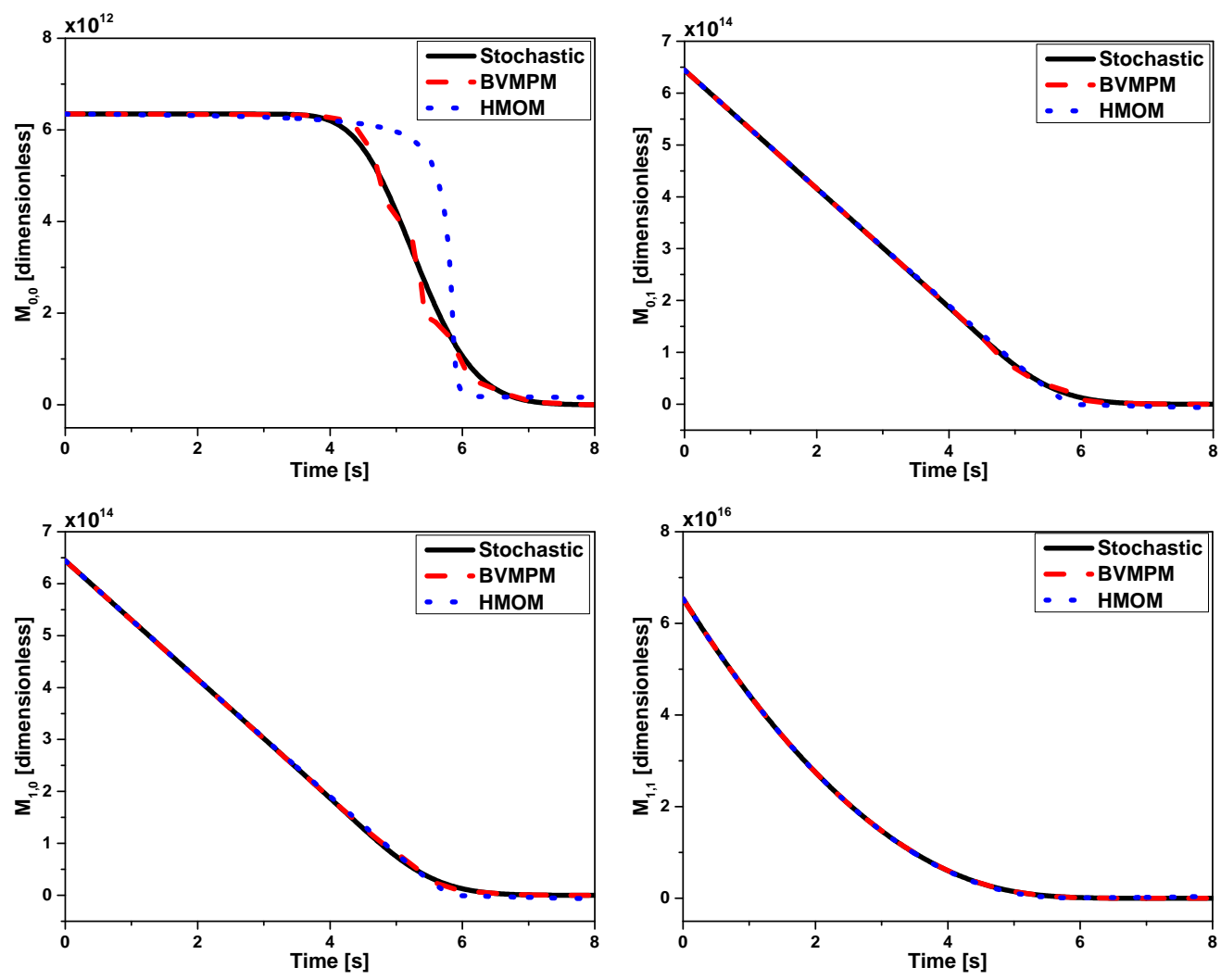

Figure 21: Comparison of $M_{0,0}$ (top left panel), $M_{0,1}$ (top right panel), $M_{1,0}$ (bottom left panel) and $M_{1,1}$ (bottom right panel) between BVMPM, HMOM and the stochastic method under all particle processes.

weighted particles are fixed at the smallest size coordinates so that the number of these weighted particles can be used to evaluate the boundary flux term due to shrinkage and the accumulation of particles at the smallest sizes due to fragmentation.

The performance of this method has been tested by comparing with the hybrid method of moments (HMOM) and the stochastic method, first for individual processes of inception, growth, shrinkage, coagulation and frag- 
mentation, then for all the processes combined. Different types of NDFs are supplied as the initial conditions. Results suggest that the weighted particles generated in BVMPM can well reproduce the behavior of particle dynamics. BVMPM exhibits very high accuracy for treating inception, growth, coagulation and fragmentation. When it comes to shrinkage, however, BVMPM shows a slight discrepancy with the stochastic solution in terms of the total number of particles. This discrepancy can be minimized by increasing the number of weighted particle sizes, $N_{1}$ or $N_{2}$. It is found that $N_{1}=4$ and $N_{2}=5$ can provide an excellent match with the stochastic solution. In general, BVMPM performs much better than HMOM in handling the shrinkage and fragmentation processes. Future work includes the application of BVMPM to real particle processes such as soot formation in flames. It remains to be seen how effective BVMPM can be for more complicated PBEs with adaptive kernels and/or free-molecular Brownian kernels.

\section{Acknowledgement}

This research is supported by the National Research Foundation, Prime Minister's Office, Singapore under its CREATE programme. 


\section{Nomenclature}

Upper-case Roman

D Eigenvectors of matrix $\mathbf{T}$

E Eigenvalues of matrix $\mathbf{T}$

$F \quad$ Source term due to fragmentation

$G$ Source term due to coagulation

H Matrix with components which are a function of conditional moments

$K_{\text {In }}$ Inception rate

$K_{\mathrm{Cg}}$ Coagulation kernel

${ }^{484} \quad K_{\mathrm{Fg}} \quad$ Fragmentation kernel

$K_{\mathrm{G}} \quad$ Growth kernel

$K_{\text {Sk }} \quad$ Shrinkage kernel

M Moment

M Matrix with components which are a function of moments

$N$ Number

$P \quad$ Fragmentation distribution function

P Matrix with components which are a function of mixed moments

$R$ Source term due to inception

R Matrix with components which are a function of conditional moments 
$S$ Source term due to shrinkage

$\mathbf{T}$ Symmetric tridiagonal matrix as a function of recursion coefficients $a$ and $b$

V Matrix with components which are a function of weighted particles

$W \quad$ Source term due to growth

Y Matrix with components which are a function of weighted marginal particles

Z Matrix with components $Z$ which are a function of the moments $M$

485

Lower-case Roman

$a, b \quad$ Recursion coefficients

$h$ Time interval

$i, j \quad$ particle size coordinate

$k, l, x, y, m, n \quad$ Indices

$r$ Recursive function

$t$ Time

Greek

$\alpha$ Particle size coordinate

$\beta \quad$ Particle size coordinate

$\gamma$ Particle size coordinate 
$\delta \quad$ Particle size change

$\eta$ Particle size coordinate

Subscripts

f Final

p Particle

0 Initial or minimum

Symbols

$\widetilde{x} \quad$ Approximation of $x$

$a \mid b \quad$ Value of $a$ given the condition of value of $b$

486

\section{Abbreviations}

BVMPM Bivariate moment projection method

ECQMOM Extended conditional quadrature method of moments

FCMOM Finite-size domain complete set of trial functions method of moments

HMOM Hybrid method of moments

PBE Population balance equation

NDF Number density function

MOM Method of moments

MOMIC Method of moments with interpolative closure

QMOM Quadrature method of moments 
PD Product difference algorithm

DQMOM Direct quadrature method of moments

EQMOM Extended quadrature method of moments

${ }_{487} \quad$ MPM Moment projection method

CQMOM Conditional quadrature method of moments

DSA Direct simulation algorithm

ODE Ordinary differential equation 


$$
W_{x, y}(M)=K_{\mathrm{G}} \sum_{i^{\prime}=i_{0}-\delta_{i}}^{\infty} \sum_{j^{\prime}=j_{0}-\delta_{j}}^{\infty}\left(i^{\prime}+\delta_{i}\right)^{x}\left(j^{\prime}+\delta_{j}\right)^{y} N\left(i^{\prime}, j^{\prime}\right)-K_{\mathrm{G}} \sum_{i=i_{0}}^{\infty} \sum_{j=j_{0}}^{\infty} i^{x} j^{y} N(i, j)
$$

501 Note that $N\left(i^{\prime}, j^{\prime}\right)=0$ for $i^{\prime}<i_{0}$ or $j^{\prime}<j_{0}$, the above equation becomes: 


$$
W_{x, y}(M)=K_{\mathrm{G}} \sum_{i^{\prime}=i_{0}}^{\infty} \sum_{j^{\prime}=j_{0}}^{\infty}\left(i^{\prime}+\delta_{i}\right)^{x}\left(j^{\prime}+\delta_{j}\right)^{y} N\left(i^{\prime}, j^{\prime}\right)-K_{\mathrm{G}} \sum_{i=i_{0}}^{\infty} \sum_{j=j_{0}}^{\infty} i^{x} j^{y} N(i, j) .
$$

502 Rewrite $i^{\prime}$ as $i$ and $j^{\prime}$ as $j$ :

$$
W_{x, y}(M)=K_{\mathrm{G}} \sum_{i=i_{0}}^{\infty} \sum_{j=j_{0}}^{\infty}\left(i+\delta_{i}\right)^{x}\left(j+\delta_{j}\right)^{y} N(i, j)-K_{\mathrm{G}} \sum_{i=i_{0}}^{\infty} \sum_{j=j_{0}}^{\infty} i^{x} j^{y} N(i, j) .
$$

503 504

505

506

507 508

Expand the first term on the right-hand side of the above equation with the binomial theorem:

$W_{x, y}(M)=K_{\mathrm{G}} \sum_{i=i_{0}}^{\infty} \sum_{j=j_{0}}^{\infty} \sum_{m=0}^{x}\left(\begin{array}{l}x \\ m\end{array}\right) i^{m} \delta_{i}^{x-m} \sum_{n=0}^{y}\left(\begin{array}{l}y \\ n\end{array}\right) j^{n} \delta_{j}^{y-n} N(i, j)-K_{\mathrm{G}} \sum_{i=i_{0}}^{\infty} \sum_{j=j_{0}}^{\infty} i^{x} j^{y} N(i, j)$.

Applying Eq. 9 to the above equation, we have:

$$
W_{x, y}(M)=K_{\mathrm{G}} \sum_{m=0}^{x} \sum_{n=0}^{y}\left(\begin{array}{l}
x \\
m
\end{array}\right)\left(\begin{array}{l}
y \\
n
\end{array}\right) \delta_{i}^{x-m} \delta_{j}^{y-n} M_{m, n}-K_{\mathrm{G}} M_{x, y} .
$$

\section{Shrinkage}

The moment source term for shrinkage is obtained by applying Eq. 9 to Eq. 4:

$$
S_{x, y}(M, N)=\sum_{i=i_{0}}^{\infty} \sum_{j=j_{0}}^{\infty} i^{x} j^{y} K_{\mathrm{Sk}}\left(N\left(i+\delta_{i}, j+\delta_{j}\right)-N(i, j)\right) .
$$


509

$$
S_{x, y}(M, N)=K_{\mathrm{Sk}} \sum_{i^{\prime}=i_{0}+\delta_{i}}^{\infty} \sum_{j^{\prime}=j_{0}+\delta_{j}}^{\infty}\left(i^{\prime}-\delta_{i}\right)^{x}\left(j^{\prime}-\delta_{j}\right)^{y} N\left(i^{\prime}, j^{\prime}\right)-K_{\mathrm{Sk}} \sum_{i=i_{0}}^{\infty} \sum_{j=j_{0}}^{\infty} i^{x} j^{y} N(i, j)
$$

510

$$
S_{x, y}(M, N)=K_{\mathrm{Sk}} \sum_{i=i_{0}+\delta_{i}}^{\infty} \sum_{j=j_{0}+\delta_{j}}^{\infty}\left(i-\delta_{i}\right)^{x}\left(j-\delta_{j}\right)^{y} N(i, j)-K_{\mathrm{Sk}} \sum_{i=i_{0}}^{\infty} \sum_{j=j_{0}}^{\infty} i^{x} j^{y} N(i, j) .
$$

511

In order to transform the terms on the right-hand side of the above equation into moments, they are rewritten as:

$$
\begin{aligned}
S_{x, y}(M, N)= & K_{\mathrm{Sk}} \sum_{i=i_{0}}^{\infty} \sum_{j=j_{0}}^{\infty}\left(i-\delta_{i}\right)^{x}\left(j-\delta_{j}\right)^{y} N(i, j)-K_{\mathrm{Sk}} \sum_{j=j_{0}}^{\infty} \sum_{i=i_{0}}^{i_{0}+\delta_{i}-1}\left(i-\delta_{i}\right)^{x}\left(j-\delta_{j}\right)^{y} N(i, j) \\
& -K_{\mathrm{Sk}} \sum_{i=i_{0}}^{\infty} \sum_{j=j_{0}}^{j_{0}+\delta_{j}-1}\left(i-\delta_{i}\right)^{x}\left(j-\delta_{j}\right)^{y} N(i, j) \\
& +K_{\mathrm{Sk}} \sum_{i=i_{0}}^{i_{0}+\delta_{i}-1} \sum_{j=j_{0}}^{j_{0}+\delta_{j}-1}\left(i-\delta_{i}\right)^{x}\left(j-\delta_{j}\right)^{y} N(i, j)-K_{\mathrm{Sk}} \sum_{i=i_{0}}^{\infty} \sum_{j=j_{0}}^{\infty} i^{x} j^{y} N(i, j) .
\end{aligned}
$$

13

(14)

tort

16

17

Assume $i^{\prime}=i+\delta_{i}$ and $j^{\prime}=j+\delta_{j}$, the above equation becomes:

Rewrite $i^{\prime}$ as $i$ and $j^{\prime}$ as $j$ : 


$$
\begin{aligned}
S_{x, y}(M, N)= & K_{\mathrm{Sk}} \sum_{i=i_{0}}^{\infty} \sum_{j=j_{0}}^{\infty} \sum_{m=0}^{x}\left(\begin{array}{l}
x \\
m
\end{array}\right) i^{m}\left(-\delta_{i}\right)^{x-m} \sum_{n=0}^{y}\left(\begin{array}{l}
y \\
n
\end{array}\right) j^{n}\left(-\delta_{j}\right)^{y-n} N(i, j) \\
& -K_{\mathrm{Sk}} \sum_{j=j_{0}}^{\infty} \sum_{i=i_{0}}^{i_{0}+\delta_{i}-1}\left(i-\delta_{i}\right)^{x}\left(j-\delta_{j}\right)^{y} N(i, j)-K_{\mathrm{Sk}} \sum_{i=i_{0}}^{\infty} \sum_{j=j_{0}}^{j_{0}+\delta_{j}-1}\left(i-\delta_{i}\right)^{x}\left(j-\delta_{j}\right)^{y} N(i, j) \\
& +K_{\mathrm{Sk}} \sum_{i=i_{0}}^{i_{0}+\delta_{i}-1} \sum_{j=j_{0}}^{j_{0}+\delta_{j}-1}\left(i-\delta_{i}\right)^{x}\left(j-\delta_{j}\right)^{y} N(i, j)-K_{\mathrm{Sk}} \sum_{i=i_{0}}^{\infty} \sum_{j=j_{0}}^{\infty} i^{x} j^{y} N(i, j) .
\end{aligned}
$$

Applying Eq. 9 to the above equation, we obtain:

$$
\begin{aligned}
S_{x, y}(M, N)= & K_{\mathrm{Sk}} \sum_{m=0}^{x} \sum_{n=0}^{y}\left(\begin{array}{c}
x \\
m
\end{array}\right)\left(\begin{array}{c}
y \\
n
\end{array}\right)\left(-\delta_{i}\right)^{x-m}\left(-\delta_{j}\right)^{y-n} M_{m, n} \\
& -K_{\mathrm{Sk}} \sum_{j=j_{0}}^{\infty} \sum_{i=i_{0}}^{i_{0}+\delta_{i}-1}\left(i-\delta_{i}\right)^{x}\left(j-\delta_{j}\right)^{y} N_{i, j}-K_{\mathrm{Sk}} \sum_{i=i_{0}}^{\infty} \sum_{j=j_{0}}^{j_{0}+\delta_{j}-1}\left(i-\delta_{i}\right)^{x}\left(j-\delta_{j}\right)^{y} N_{i, j} \\
& +K_{\mathrm{Sk}} \sum_{i=i_{0}}^{i_{0}+\delta_{i}-1} \sum_{j=j_{0}}^{j_{0}+\delta_{j}-1}\left(i-\delta_{i}\right)^{x}\left(j-\delta_{j}\right)^{y} N_{i, j}-K_{\mathrm{Sk}} M_{x, y} .
\end{aligned}
$$

It can be seen that the numbers of particles at the smallest size coordinates are needed to evaluate the second, third and forth terms on the right-hand side of the above equation.

\section{Coagulation}

Applying Eq. 9 to Eq. 5, the moment source term for coagulation is obtained: 


$$
\begin{aligned}
G_{x, y}(M)= & \frac{1}{2} K_{\mathrm{Cg}} \sum_{i=i_{0}}^{\infty} \sum_{j=j_{0}}^{\infty} i^{x} j^{y} \sum_{i^{\prime}=i_{0}}^{i} \sum_{j^{\prime}=j_{0}}^{j} N\left(i-i^{\prime}, j-j^{\prime}\right) N\left(i^{\prime}, j^{\prime}\right) \\
& -K_{\mathrm{Cg}} \sum_{i=i_{0}}^{\infty} \sum_{j=j_{0}}^{\infty} i^{x} j^{y} \sum_{i^{\prime}=i_{0}}^{\infty} \sum_{j^{\prime}=j_{0}}^{\infty} N(i, j) N\left(i^{\prime}, j^{\prime}\right) .
\end{aligned}
$$

525

Assume $w=i-i^{\prime}$ and $v=j-j^{\prime}$ :

$$
\begin{aligned}
G_{x, y}(M)= & \frac{1}{2} K_{\mathrm{Cg}} \sum_{w+i^{\prime}=i_{0}}^{\infty} \sum_{v+j^{\prime}=j_{0}}^{\infty} \sum_{i^{\prime}=i_{0}}^{w+i^{\prime}} \sum_{j^{\prime}=j_{0}}^{v+j^{\prime}}\left(w+i^{\prime}\right)^{x}\left(v+j^{\prime}\right)^{y} N(w, v) N\left(i^{\prime}, j^{\prime}\right) \\
& -K_{\mathrm{Cg}} \sum_{i=i_{0}}^{\infty} \sum_{j=j_{0}}^{\infty} i^{x} j^{y} N(i, j) \sum_{i^{\prime}=i_{0}}^{\infty} \sum_{j^{\prime}=j_{0}}^{\infty} N\left(i^{\prime}, j^{\prime}\right)
\end{aligned}
$$

526

Note that $N(w, v)=0$ for $w<i_{0}$ or $v<j_{0}$, the above equation becomes:

$$
\begin{aligned}
G_{x, y}(M)= & \frac{1}{2} K_{\mathrm{Cg}} \sum_{w=i_{0}}^{\infty} \sum_{v=j_{0}}^{\infty} \sum_{i^{\prime}=i_{0}}^{\infty} \sum_{j^{\prime}=j_{0}}^{\infty}\left(w+i^{\prime}\right)^{x}\left(v+j^{\prime}\right)^{y} N(w, v) N\left(i^{\prime}, j^{\prime}\right) \\
& -K_{\mathrm{Cg}} \sum_{i=i_{0}}^{\infty} \sum_{j=j_{0}}^{\infty} i^{x} j^{y} N(i, j) \sum_{i^{\prime}=i_{0}}^{\infty} \sum_{j^{\prime}=j_{0}}^{\infty} N\left(i^{\prime}, j^{\prime}\right)
\end{aligned}
$$

527 
529

Apply Eq. 9 to the above equation, we have:

$$
G_{x, y}(M)=\frac{1}{2} K_{\mathrm{Cg}} \sum_{m=0}^{x} \sum_{n=0}^{y}\left(\begin{array}{l}
x \\
m
\end{array}\right)\left(\begin{array}{l}
y \\
n
\end{array}\right) M_{m, n} M_{x-m, y-n}-K_{\mathrm{Cg}} M_{x, y} M_{0,0}
$$

530

531 532

$$
\begin{aligned}
F_{x, y}(M, N)= & \sum_{i^{\prime}=i_{0}}^{\infty} \sum_{j^{\prime}=j_{0}}^{\infty} \sum_{i=i_{0}}^{i^{\prime}} \sum_{j=j_{0}}^{j^{\prime}} i^{x} j^{y} K_{\mathrm{Fg}}\left(i^{\prime}, j^{\prime}\right) P\left(i, j \mid i^{\prime}, j^{\prime}\right) N\left(i^{\prime}, j^{\prime}\right) \\
& -\sum_{i=i_{0}}^{\infty} \sum_{j=j_{0}}^{\infty} i^{x} j^{y} K_{\mathrm{Fg}}(i, j) N(i, j) .
\end{aligned}
$$

${ }_{534}$ Note that $K_{\mathrm{Fg}}(i, j)=0$ for $i<2 i_{0}$ or $j<2 j_{0}$ otherwise the total particle 535 


$$
\begin{aligned}
F_{x, y}(M, N)= & \sum_{i^{\prime}=2 i_{0}}^{\infty} \sum_{j^{\prime}=2 j_{0}}^{\infty} K_{\mathrm{Fg}} N\left(i^{\prime}, j^{\prime}\right) \sum_{i=i_{0}}^{i^{\prime}} \sum_{j=j_{0}}^{j^{\prime}} i^{x} j^{y} P\left(i, j \mid i^{\prime}, j^{\prime}\right) \\
& -\sum_{i=2 i_{0}}^{\infty} \sum_{j=2 j_{0}}^{\infty} i^{x} j^{y} K_{\mathrm{Fg}} N(i, j) .
\end{aligned}
$$

536 Applying Eq 8 into the above equation:

$$
\begin{aligned}
F_{x, y}(M, N)= & \sum_{i^{\prime}=2 i_{0}}^{\infty} \sum_{j^{\prime}=2 j_{0}}^{\infty} K_{\mathrm{Fg}} N\left(i^{\prime}, j^{\prime}\right)\left(i_{0}^{x} j_{0}^{y}+\left(i^{\prime}-i_{0}\right)^{x}\left(j^{\prime}-j_{0}\right)^{y}\right) \\
& -\sum_{i=2 i_{0}}^{\infty} \sum_{j=2 j_{0}}^{\infty} K_{\mathrm{Fg}} i^{x} j^{y} N(i, j)
\end{aligned}
$$

${ }_{537}$ Let $i^{\prime}=i, j^{\prime}=j$ and rewrite the above equation as:

$$
F_{x, y}(M, N)=\sum_{i=2 i_{0}}^{\infty} \sum_{j=2 j_{0}}^{\infty} K_{\mathrm{Fg}} N(i, j)\left(i_{0}^{x} j_{0}^{y}+\left(i-i_{0}\right)^{x}\left(j-j_{0}\right)^{y}-i^{x} j^{y}\right)
$$

${ }_{538}$ To transform the terms on the right-hand side of the above equation into moments, the above equation is rewritten as: 


$$
\begin{aligned}
F_{x, y}(M, N)= & \sum_{i=i_{0}}^{\infty} \sum_{j=j_{0}}^{\infty} K_{\mathrm{Fg}} N(i, j)\left(i_{0}^{x} j_{0}^{y}+\left(i-i_{0}\right)^{x}\left(j-j_{0}\right)^{y}-i^{x} j^{y}\right) \\
& -\sum_{i=i_{0}}^{2 i_{0}-1} \sum_{j=j_{0}}^{\infty} K_{\mathrm{Fg}} N(i, j)\left(i_{0}^{x} j_{0}^{y}+\left(i-i_{0}\right)^{x}\left(j-j_{0}\right)^{y}-i^{x} j^{y}\right) \\
& -\sum_{i=i_{0}}^{\infty} \sum_{j=j_{0}}^{2 j_{0}-1} K_{\mathrm{Fg}} N(i, j)\left(i_{0}^{x} j_{0}^{y}+\left(i-i_{0}\right)^{x}\left(j-j_{0}\right)^{y}-i^{x} j^{y}\right) \\
& +\sum_{i=i_{0}}^{2 i_{0}-1} \sum_{j=j_{0}}^{2 j_{0}-1} K_{\mathrm{Fg}} N(i, j)\left(i_{0}^{x} j_{0}^{y}+\left(i-i_{0}\right)^{x}\left(j-j_{0}\right)^{y}-i^{x} j^{y}\right) .
\end{aligned}
$$

$$
\begin{aligned}
F_{x, y}(M, N)= & \sum_{i=i_{0}}^{\infty} \sum_{j=j_{0}}^{\infty} K_{\mathrm{Fg}} N(i, j)\left(i_{0}^{x} j_{0}^{y}-i^{x} j^{y}+\sum_{m=0}^{x}\left(\begin{array}{l}
x \\
m
\end{array}\right) i^{m}\left(-i_{0}\right)^{x-m} \sum_{n=0}^{y}\left(\begin{array}{l}
y \\
n
\end{array}\right) j^{n}\left(-j_{0}\right)^{y-n}\right) \\
& -\sum_{i=i_{0}}^{2 i_{0}-1} \sum_{j=j_{0}}^{\infty} K_{\mathrm{Fg}} N(i, j)\left(i_{0}^{x} j_{0}^{y}+\left(i-i_{0}\right)^{x}\left(j-j_{0}\right)^{y}-i^{x} j^{y}\right) \\
& -\sum_{i=i_{0}}^{\infty} \sum_{j=j_{0}}^{2 j_{0}-1} K_{\mathrm{Fg}} N(i, j)\left(i_{0}^{x} j_{0}^{y}+\left(i-i_{0}\right)^{x}\left(j-j_{0}\right)^{y}-i^{x} j^{y}\right) \\
& +\sum_{i=i_{0}}^{2 i_{0}-1} \sum_{j=j_{0}}^{2 j_{0}-1} K_{\mathrm{Fg}} N(i, j)\left(i_{0}^{x} j_{0}^{y}+\left(i-i_{0}\right)^{x}\left(j-j_{0}\right)^{y}-i^{x} j^{y}\right) .
\end{aligned}
$$


546

Applying Eq. 9 to the above equation, we obtain:

$$
\begin{aligned}
F_{x, y}(M, N)= & K_{\mathrm{Fg}} \sum_{m=0}^{x} \sum_{n=0}^{y}\left(\begin{array}{c}
x \\
m
\end{array}\right)\left(\begin{array}{l}
y \\
n
\end{array}\right)\left(-i_{0}\right)^{x-m}\left(-j_{0}\right)^{y-n} M_{m, n}+K_{\mathrm{Fg}} i_{0}^{x} j_{0}^{y} M_{0,0}-K_{\mathrm{Fg}} M_{x, y} \\
& -K_{\mathrm{Fg}} \sum_{j=j_{0}}^{\infty} \sum_{i=i_{0}}^{2 i_{0}-1}\left(\left(i-i_{0}\right)^{x}\left(j-j_{0}\right)^{y}+i_{0}^{x} j_{0}^{y}-i^{x} j^{y}\right) N_{i, j} \\
& -K_{\mathrm{Fg}} \sum_{i=i_{0}}^{\infty} \sum_{j=j_{0}}^{2 j_{0}-1}\left(\left(i-i_{0}\right)^{x}\left(j-j_{0}\right)^{y}+i_{0}^{x} j_{0}^{y}-i^{x} j^{y}\right) N_{i, j} \\
& +K_{\mathrm{Fg}} \sum_{i=i_{0}}^{2 i_{0}-1} \sum_{j=j_{0}}^{2 j_{0}-1}\left(\left(i-i_{0}\right)^{x}\left(j-j_{0}\right)^{y}+i_{0}^{x} j_{0}^{y}-i^{x} j^{y}\right) N_{i, j} . \quad \text { (A.26) }
\end{aligned}
$$

547 It can be seen that the numbers of particles at the smallest size coordinates

\section{Appendix B. 1-D Blumstein and Wheeler algorithm}

This algorithm is used to determine the sizes and corresponding number of weighted particles to approximate the univariate NDF from the empirical moments. 


\section{Algorithm 2: 1-D Blumstein and Wheeler algorithm.}

Input: The empirical moments $\widetilde{M}_{x}$ for $x=0,1, \ldots, 2 N_{1}-2$.

Output: The particle size $\alpha_{k}$ and the corresponding number of weighted particles $\widetilde{N}_{\alpha_{k}}$ for $k=1,2, \ldots, N_{1}$.

for $N_{p}=2$ to $N_{1}$ do

Determine the elements of the first row of matrix $\mathbf{Z}: Z_{1, j}=\widetilde{M}_{j-1}$ for $j=1, \ldots, 2 N_{\mathrm{p}}-1$.

For $a_{1}=\widetilde{M}_{1} / \widetilde{M}_{0}$ and $b_{1}=0$, determine the recursion coefficients $a_{i}$ and $b_{i}$ :

for $i=2$ to $N_{p}$ do

for $j=i$ to $2 N_{p}-1$ do

The elements of $\mathbf{Z}$ must satisfy the following recursion relation:

$$
Z_{i, j}=Z_{i-1, j+1}-a_{i-1} Z_{i-1, j}-b_{i-1} Z_{i-1, j}
$$

If $Z_{i, i}<M_{\min }$ or $Z_{i-1, i-1}<M_{\min }$, exit the main loop. Otherwise

$$
a_{i}=\frac{Z_{i, i+1}}{Z_{i, i}}-\frac{Z_{i-1, i}}{Z_{i-1, i-1}} ; \quad b_{i}=\frac{Z_{i, i}}{Z_{i-1, i-1}} .
$$

For $r_{1}=1 /\left(i_{0}-a_{1}\right)$ where $i_{0}$ is the smallest particle size, determine the recursion function:

$$
r_{i}=1 /\left(i_{0}-a_{i}-b_{i} r_{i-1}\right) \quad i=2, \ldots, N_{\mathrm{p}}-1 .
$$

As we fix the smallest particle size, replace $a_{N_{\mathrm{p}}}$ with:

$$
a_{N_{\mathrm{p}}}=i_{0}-b_{N_{\mathrm{p}}} r_{N_{\mathrm{p}}-1}
$$

Construct a symmetric tridiagonal matrix $\mathbf{T}$ with $a_{i}$ as the diagonal and the square roots of $b_{i}$ as the co-diagonal:

$$
\mathbf{T}=\left[\begin{array}{ccccc}
a_{1} & -\sqrt{b_{2}} & 0 & \cdots & 0 \\
-\sqrt{b_{2}} & a_{2} & -\sqrt{b_{3}} & \cdots & 0 \\
0 & -\sqrt{b_{3}} & a_{3} & \cdots & 0 \\
\vdots & \vdots & \vdots & \ddots & \vdots \\
0 & 0 & 0 & \cdots & a_{\mathrm{N}_{p}}
\end{array}\right]
$$

Solve for the eigenvalues $\mathbf{E}$ and eigenvectors $\mathbf{D}$ of matrix $\mathbf{T}$ :

$$
[\mathbf{E}, \mathbf{D}]=\operatorname{eig}(\mathbf{T})
$$

If any diagornal element of matrix $\mathbf{E}$ is smaller than $i_{0}$ or any element in the first row of matrix $\mathbf{T}$ is negative, exit the main loop and adopt the weighted particles obtained in the last loop as the final output.

Otherwise determine $\alpha_{k}$ and $\widetilde{N}_{\alpha_{k}}$ by:

$$
\alpha_{k}=\mathbf{E}(k, k), \quad \widetilde{N}_{\alpha_{k}}=\widetilde{M}_{0} \mathbf{D}(1, k)^{2} .
$$




\section{${ }_{555}$ Appendix C. 2-D Blumstein and Wheeler algorithm}

556 This algorithm is used to generate the weighted particles to approximate 557 the bivariate NDF from the empirical moments. It involves multiple appli${ }_{558}$ cations of 1-D Blumstein and Wheeler algorithm. 


\section{Algorithm 3: 2-D Blumstein and Wheeler algorithm.}

Input: The empirical moments $\widetilde{M}_{x, y}$ for $x=0,1, \ldots, 2 N_{1}-2$ and

$$
y=0,1, \ldots, 2 N_{2}-2 .
$$

Output: The weighted particle internal coordinates $\left(\alpha_{k}, \beta_{l \mid k}\right)$ and the corresponding numbers $\widetilde{N}_{\alpha_{k}}$ and $\widetilde{N}_{\beta_{l \mid k}}$ for $k=1,2, \ldots, N_{1}$ and $l=1,2, \ldots, N_{2}$.

Use the marginal moments $\widetilde{M}_{x, 0}\left(x=0, \ldots, 2 N_{1}-2\right)$ to determine $\alpha_{k}$ and $\widetilde{N}_{\alpha_{k}}\left(k=1, \ldots, N_{1}\right)$ with the 1 -D Blumstein and Wheeler algorithm.

Create a $N_{1} \times N_{1}$ matrix $\mathbf{Y}$ and a $N_{1} \times\left(2 N_{2}-1\right)$ matrix $\mathbf{M}$ with zeros in all elements.

for $i=1$ to $N_{1}$ do

for $j=1$ to $N_{1}$ do

Determine the elements of $\mathbf{Y}$ with the weighted marginal particles:

$Y_{i, j}=\alpha_{j}^{i-1} \widetilde{N}_{\alpha_{j}}$.

for $i=1$ to $N_{1}$ do

for $j=1$ to $2 N_{2}-1$ do

Determine the elements of $\mathbf{M}$ with the empirical moments:

$M_{i, j}=\widetilde{M}_{i-1, j}$.

Create a $N_{1} \times\left(2 N_{2}-1\right)$ matrix $\mathbf{H}$ with the elements in the first column are 1 and the others are determined by

$\mathbf{H}\left(1: N_{1}, 2: 2 N_{2}-1\right)=\mathbf{Y}^{-1} \mathbf{M}$.

for $k=1$ to $N_{1}$ do

With $\mathbf{H}\left(k, 1: 2 N_{2}-1\right)$, use the 1-D Blumstein and Wheeler

algorithm to determine the conditional weighted particles: $\beta_{l \mid k}$ and $\tilde{N}_{\beta_{l \mid k}}$. 
[1] A. D. Randolph, M. A. Larson, Theory of Particulate Processes: Analysis and Techniques of Continuous Crystallization, Academic Press, San Diego, 1988.

[2] M. D. Smooke, C. S. McEnally, L. D. Pfefferle, R. J. Hall, M. B. Colket, Computational and experimental study of soot formation in a coflow, laminar diffusion flame, Combust. Flame. 117 (1999) 117-139. doi:10.1016/S0010-2180(98)00096-0.

[3] J. Singh, M. Balthasar, M. Kraft, W. Wagner, Stochastic modeling of soot particle size and age distributions in laminar premixed flames, Proc. Combust. Inst. 30 (2005) 1457-1465. doi:10.1016/j.proci.2004.08.120.

[4] E. K. Y. Yapp, R. I. A. Patterson, J. Akroyd, S. Mosbach, E. M. Adkins, M. J. Houston, M. Kraft, Numerical simulation and parametric sensitivity study of optical band gap in a laminar coflow ethylene diffusion flame, Combust. Flame 167 (2016) 320-334. doi:10.1016/j.combustflame.2016.01.033.

[5] S. Rigopoulos, Population balance modelling of polydispersed particles in reactive flows, Prog. Energ. Combust. 36 (2010) 412-443. doi:10.1016/j.pecs.2009.12.001.

[6] J. Akroyd, A. J. Smith, R. Shirley, L. R. McGlashan, M. Kraft, A coupled CFD-population balance approach for nanoparticle synthesis in turbulent reacting flows, Chem. Eng. Sci. 66 (2011) 3792-3805. doi:10.1016/j.ces.2011.05.006. 
[7] S. Shekar, W. J. Menz, A. J. Smith, M. Kraft, W. Wagner, On a multivariate population balance model to describe the structure and composition of silica nanoparticles, Comput. Chem. Eng. 43 (2012) 130-147. doi:10.1016/j.compchemeng.2012.04.010.

[8] S. Shekar, A. J. Smith, W. J. Menz, M. Sander, M. Kraft, A multidimensional population balance model to describe the aerosol synthesis of silica nanoparticles, J. Aerosol Sci. 44 (2012) 83-98. doi:10.1016/j.jaerosci.2011.09.004.

[9] D. Grosschmidt, H. Bockhorn, M. Goodson, M. Kraft, Two approaches to the simulation of silica particle synthesis, Proc. Combust. Inst. 29 (2002) 1039-1046. doi:10.1016/S1540-7489(02)80131-6.

[10] D. Ramkrishna, Population Balances: Theory and Applications to Particulate Systems in Engineering, Academic Press, New York, 2000.

[11] M. Kraft, Modelling of particulate processes, KONA Powder Part. J. 23 (2005) 18-35. doi:10.14356/kona.2005007.

[12] M. Balthasar, M. Kraft, A stochastic approach to calculate the particle size distribution function of soot particles in laminar premixed flames, Combust. Flame. 133 (2003) 289-298. doi:10.1016/S00102180(03)00003-8.

[13] R. I. A. Patterson, W. Wagner, M. Kraft, Stochastic weighted particle methods for population balance equations, J. Comput. Phys. 230 (2011) 7456-7472. doi:10.1016/j.jcp.2011.06.011. 
[14] M. J. Hounslow, R. L. Ryall, V. R. Marshall, Discretized population balance for nucleation, growth and aggregation, AIChE J. 34 (1988) 1821-1832. doi:10.1002/aic.690341108.

[15] M. J. Hounslow, A discretized population balance for continuous systems at steady state, AIChE J. 36 (1990) 106-116. doi:10.1002/aic.690360113.

[16] S. Kumar, D. Ramkrishna, On the solution of population balance equations by discretization-I. A fixed pivot technique, Chem. Eng. Sci. 51 (1996) 1311-1332. doi:10.1016/0009-2509(96)88489-2.

[17] S. Kumar, D. Ramkrishna, On the solution of population balance equations by discretization-II. A moving pivot technique., Chem. Eng. Sci. 51 (1996) 1333-1342. doi:10.1016/0009-2509(95)00355-X.

[18] J. C. Barrett, N. A. Webb, A comparison of some approximate methods for solving the aerosol general dynamic equation, J. Aerosol Sci. 29 (1998) 31-39. doi:10.1016/S0021-8502(97)00455-2.

[19] M. Frenklach, Method of moments with interpolative closure, Chem. Eng. Sci. 57 (2002) 2229-2239. doi:10.1016/S0009-2509(02)00113-6.

[20] M. Frenklach, S. J. Harris, Aerosol dynamics modeling using the method of moments, J. Colloid Interface Sci. 118 (1987) 252-261. doi:10.1016/0021-9797(87)90454-1.

[21] M. Balthasar, M. Frenklach, Detailed kinetic modeling of soot aggregate formation in laminar premixed flames, Combust. Flame 140 (2005) 130145. doi:10.1016/j.combustflame.2004.11.004. 
[22] A. Kazakov, M. Frenklach, Dynamic modeling of soot particle coagulation and aggregation: implementation with the method of moments and application to high-pressure laminar premixed flames, Combust. Flame 114 (1998) 484-501. doi:10.1016/S0010-2180(97)00322-2.

[23] R. McGraw, Description of aerosol dynamics by the quadrature method of moments, Aerosol Sci. Technol. 27 (1997) 255-265. doi:10.1080/02786829708965471.

[24] D. L. Marchisio, R. D. Vigil, R. O. Fox, Quadrature method of moments for aggregation-breakage processes, J. Colloid Interface Sci. 258 (2003) 322-334. doi:10.1016/S0021-9797(02)00054-1.

[25] D. L. Marchisio, J. T. Pikturna, R. O. Fox, R. D. Vigil, A. A. Barresi, Quadrature method of moments for population-balance equations, AIChE. J. 49 (2003) 1266-1276. doi:10.1002/aic.690490517.

[26] D. L. Marchisio, R. D. Vigil, R. O. Fox, Implementation of the quadrature method of moments in CFD codes for aggregation-breakage problems, Chem. Eng. Sci. 58 (2003) 3337-3351. doi:10.1016/S00092509(03)00211-2.

[27] R. G. Gordon, Error bounds in equilibrium statistical mechanics, J. Math. Phys. 9 (1968) 655-663. doi:10.1063/1.1664624.

[28] D. L. Marchisio, R. O. Fox, Solution of population balance equations using the direct quadrature method of moments, J. Aerosol Sci. 36 (2005) 43-73. doi:10.1016/j.jaerosci.2004.07.009. 
[29] S. Salenbauch, A. Cuoci, A. Frassoldati, C. Saggese, T. Faravelli, Modeling soot formation in premixed flames using an extended conditional quadrature method of moments, Combust. Flame 162 (2015) 2529-2543. doi:10.1016/j.combustflame.2015.03.002.

[30] M. Strumendo, H. Arastoopour, Solution of PBE by MOM in finite size domains, Chem. Eng. Sci. 63 (2008) 2624-2640. doi:10.1016/j.ces.2008.02.010.

[31] C. Yuan, F. Laurent, R. O. Fox, An extended quadrature method of moments for population balance equations, J. Aerosol Sci. 51 (2012) 1-23. doi:10.1016/j.jaerosci.2012.04.003.

[32] E. Madadi-Kandjani, A. Passalacqua, An extended quadrature-based moment method with log-normal kernel density functions, Chem. Eng. Sci. 131 (2015) 323-339. doi:10.1016/j.ces.2015.04.005.

[33] R. B. Diemer, J. H. Olson, A moment methodology for coagulation and breakage problems: part 2-moment models and distribution reconstruction, Chem. Eng. Sci. 57 (2002) 2211-2228. doi:10.1016/S00092509(02)00112-4.

[34] A. Falola, A. Borissova, X. Z. Wang, Extended method of moment for general population balance models including size dependent growth rate, aggregation and breakage kernels, Comp. Chem. Eng. 56 (2013) 1-11. doi:10.1016/j.compchemeng.2013.04.017.

[35] A. Wick, T. T. Nguyen, F. Laurent, R. O. Fox, H. Pitsch, Modeling soot 
oxidation with the Extended Quadrature Method of Moments, Proc. Combust. Inst. 36 (2017) 789-797. doi:10.1016/j.proci.2016.08.004.

[36] H. M. Hulburt, S. Katz, Some problems in particle technology: A statistical mechanical formulation, Chem. Eng. Sci. 19 (1964) 555-574. doi:10.1016/0009-2509(64)85047-8.

[37] D. L. Wright, R. McGraw, D. E. Rosner, Bivariate extension of the quadrature method of moments for modeling simultaneous coagulation and sintering of particle populations, J. Colloid Interface Sci. 236 (2001) 242-251. doi:10.1006/jcis.2000.7409.

[38] S. Qamar, S. Noor, Q. u. Ain, A. Seidel-Morgenstern, Bivariate extension of the quadrature method of moments for batch crystallization models, Ind. Eng. Chem. Res. 49 (2010) 11633-11644. doi:10.1021/ie101108s.

[39] C. Yuan, R. O. Fox, Conditional quadrature method of moments for kinetic equations, J. Comput. Phys. 230 (2011) 8216-8246. doi:10.1016/j.jcp.2011.07.020.

[40] M. Mehta, V. Raman, R. O. Fox, On the role of gas-phase and surface chemistryin the production of titania nanoparticles in turbulent flames, Chem. Eng. Sci. 104 (2013) 1003-1018. doi:10.1016/j.ces.2013.10.039.

[41] J. C. Cheng, R. O. Fox, Kinetic modeling of nanoprecipitation using CFD coupled with a population balance, Ind. Eng. Chem. Res. 49 (2010) 10651-10662. doi:10.1021/ie100558n.

[42] S. Salenbauch, M. Sirignano, D. L. Marchisio, M. Pollack, A. D'Anna, C. Hasse, Detailed particle nucleation modeling in 
a sooting ethylene flame using a conditional quadrature method of moments (CQMOM), Proc. Combust. Inst. 36 (2017) 771-779. doi:10.1016/j.proci.2016.08.003.

[43] M. E. Mueller, G. Blanquart, H. Pitsch, Hybrid method of moments for modelling soot formation and growth, Combust. Flame. 156 (2009) 1143-1155. doi:10.1016/j.combustflame.2009.01.025.

[44] M. E. Mueller, G. Blanquart, H. Pitsch, Modeling the oxidation-induced fragmentation of soot aggregates in laminar flames, Proc. Combust. Inst. 33 (2011) 667-674. doi:10.1016/j.proci.2010.06.036.

[45] S. Wu, E. K. Y. Yapp, J. Akroyd, S. Mosbach, R. Xu, W. Yang, M. Kraft, A moment projection method for population balance dynamics with a shrinkage term, J. Comput. Phys. 330 (2017) 960-980. doi:10.1016/j.jcp.2016.10.030.

[46] S. Wu, E. K. Y. Yapp, J. Akroyd, S. Mosbach, R. Xu, W. Yang, M. Kraft, Extension of moment projection method to the fragmentation process, J. Comput. Phys. 335 (2017) 516-534. doi:10.1016/j.jcp.2017.01.045.

[47] D. L. Wright Jr, Numerical advection of moments of the particle size distribution in Eulerian models, J. Aerosol Sci. 38 (2007) 352-369. doi:10.1016/j.jaerosci.2006.11.011.

[48] V. Vikas, Z. J. Wang, A. Passalacqua, R. O. Fox, Realizable high-order finite-volume schemes for quadrature-based moment methods, J. Comput. Phys. 230 (2011) 5328-5352. doi:10.1016/j.jcp.2011.03.038. 
716

717

718

719

720

721

722

[49] R. M. Ziff, New solutions to the fragmentation equation, J. Phys. A Math. Gen. 24 (1991) 2821-2828. doi:10.1088/0305-4470/24/12/020.

[50] E. D. McGrady, R. M. Ziff, "Shattering" transition in fragmentation, Phys. Rev. Lett. 58 (1987) 892-895. doi:10.1103/PhysRevLett.58.892.

[51] C. Blumstein, J. C. Wheeler, Modified-moments method: applications to harmonic solids, Phys. Rev. B. 8 (1973) 1764-1776. doi:10.1103/PhysRevB.8.1764. 